\title{
Genome-wide identification of the NLR gene family in Haynaldia villosa by SMRT-RenSeq
}

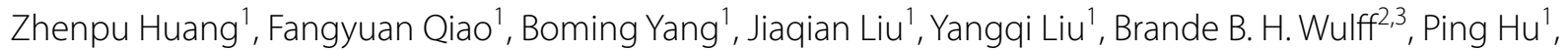
Zengshuai Lv' ${ }^{1}$, Ruiqi Zhang ${ }^{1}$, Peidu Chen ${ }^{1}$, Liping Xing ${ }^{1 *}$ and Aizhong Cao ${ }^{1 *}$

\begin{abstract}
Background: Nucleotide-binding and leucine-rich repeat (NLR) genes have attracted wide attention due to their crucial role in protecting plants from pathogens. SMRT-RenSeq, combining PacBio sequencing after resistance gene enrichment sequencing (RenSeq), is a powerful method for selectively capturing and sequencing full-length NLRs. Haynaldia villosa, a wild grass species with a proven potential for wheat improvement, confers resistance to multiple diseases. So, genome-wide identification of the NLR gene family in Haynaldia villosa by SMRT-RenSeq can facilitate disease resistance genes exploration.

Results: In this study, SMRT-RenSeq was performed to identify the genome-wide NLR complement of H. villosa. In total, 1320 NLRs were annotated in 1169 contigs, including 772 complete NLRs. All the complete NLRs were phylogenetically analyzed and 11 main clades with special characteristics were derived. NLRs could be captured with high efficiency when aligned with cloned $\mathrm{R}$ genes, and cluster expansion in some specific gene loci was observed. The physical location of NLRs to individual chromosomes in H. villosa showed a perfect homoeologous relationship with group 1, 2, 3, 5 and 6 of other Triticeae species, however, NLRs physically located on 4VL were largely in silico predicted to be located on the homoeologous group 7. Fifteen types of integrated domains (IDs) were integrated in 52 NLRs, and Kelch and B3 NLR-IDs were found to have expanded in H. villosa, while DUF948, NAM-associated and PRT_C were detected as unique integrated domains implying the new emergence of NLR-IDs after $\mathrm{H}$. villosa diverged from other species.

Conclusion: SMRT-RenSeq is a powerful tool to identify NLR genes from wild species using the baits of the evolutionary related species with reference sequences. The availability of the NLRs from $H$. villosa provide a valuable library for $R$ gene mining and transfer of disease resistance into wheat.
\end{abstract}

Keywords: NLR, Haynaldia villosa, SMRT-RenSeq, Disease resistance, Genomics

\section{Background}

Plants have evolved comprehensive mechanisms to protect themselves from attack by pests and pathogens [18]. The first level of protection is provided by the physical barriers imposed by the plant surface, a type of resistance

\footnotetext{
*Correspondence: xingliping@njau.edu.cn; caoaz@njau.edu.cn ${ }^{1}$ National Key Laboratory of Crop Genetics and Germplasm Enhancement, Cytogenetics Institute, Nanjing Agricultural University/CICMCP, Nanjing 210095, China

Full list of author information is available at the end of the article
}

often termed passive defense [70]. The second level of protection is induced by recognition of pathogen associated molecular patterns (PAMPs) by pattern-recognition receptors (PRRs), which are usually extracellular plasma membrane anchored; this active defense is often called PAMP-triggered immunity (PTI) [7]. The last level of protection is induced by recognition of pathogen effectors by the products of plant resistance genes usually located in the cytoplasm; this active defense is often called effector-triggered immunity (ETI) [28]. 
To date, more than 300 resistance ( $R$ genes) defined by genetics have been cloned from a wide range of plant species [36]. The majority of these $(>80 \%)$ encode intracellular immune receptors of the nucleotide-binding and leucine-rich repeat (NLR) class of genes. NLRs have also been found to induce defense responses in animals [29, 48]. Genomic reference quality assemblies now make it possible to characterize complete $N L R$ repertoires in plants. Typically, several hundred NLRs are found in a plant genome. For example, 149 NLRs were identified in Arabidopsis [50], 459 in Vitis vinifera, 330 in Populus trichocarpa [81], 319 in Glycine max [35] and 327 in Manihot esculenta [46]. Genome-wide NLR complements have also been studied in five species of Brassicaceae [88], four species of Gossypium [78], seven species of Leguminosae [89], three species of Solanaceae [60], and in several species of grasses including Sorghum bicolor, Zea mays, Brachypodium distachion, cultivated and wild Oryza species, and several Triticeae species [4, $15,41,61,69,80]$. In hexaploid bread wheat, 3400 NLRs were identified, the largest number reported thus far in a plant species [69]. The number of NLRs appears to correlate positively with the total number of genes in the genome [3], but less so with genome size. For example, more than 1000 NLRs were identified in apple $(740 \mathrm{Mb}$ genome [27];), while only 151 were detected in maize (2.1 Gb genome [66];) and 54 in Carica papaya (370 Mb genome [58];). However, higher ploidy levels does tend to correlate with a larger number of NLRs, such as observed in wheat and apple $[63,73]$.

With the recent rapid advances in bioinformatics and genomics, huge progress has been made in understanding Triticeae genomes, including more efficient and complete characterization of their NLR complements. The NLR contents have been identified in T. urartu, Ae. tauschii and T. aestivum by different researchers [22, 69]. However, due to different draft genome versions, distinct annotation pipelines with various parameters and the high sequence similarity among NLRs, the number of identified NLRs can vary widely between different studies. In those species which lack a reference genome, it is even more challenging to perform a genome-wide NLR survey. Exon capture enrichment allows the selected sequencing of an exome [54], or a specific gene family [32]. The highly conserved domains shared by different NLRs provides perfect targets for enrichment. The $R$ gene enrichment and sequencing (RenSeq) method provides a powerful and attractive tool for the identification of NLRs from plants without finished reference genome assemblies, in particular those plants with large genomes and higher ploidy levels, for example hexaploid wheat and octaploid strawberry [6]. RenSeq was first applied to identify NLRs from Solanum tuberosum, and it indicated that $\sim 80 \%$ sequence identity between NLR genes and the corresponding oligonucleotide baits was sufficient for enrichment. This pioneering use of RenSeq increased the number of annotated NLRs in potato from 438 to 772, and facilitated the genetic mapping of NLRs associated with disease resistance to poorly or previously unannotated regions of the genome [34]. MutRenSeq, combining RenSeq with mutant development, was used to clone the stem rust resistance genes $\mathrm{Sr} 22$ and $\mathrm{Sr} 45$ from hexaploid wheat [68]. More recently, association genetics combined with RenSeq (AgRenSeq) on a wild population of diploid wheat (Aegilops tauschii), AgRenSeq, facilitated the rapid identification four stem rust resistance genes [2]. The assemblies generated by RenSeq with short-read Illumina sequencing technology are, however, typically fragmented and incomplete. For example, RenSeq targeting Sr22 and Sr33 resulted in two and three contigs, respectively, with missing gaps corresponding largely to the introns. RenSeq combined with long read sequencing, such as PacBio single-molecule real-time (SMRT) or Oxford Nanopore Technology, mitigates these limitations by generating more complete assemblies including NLRs with novel integrated domains [19, 76]. SMRTRenSeq facilitated the cloning of the Phytophothora infestans resistance gene Rpi-amr3i from the wild potato relative Solanum americanum [76], the Potato Virus $Y$ resistance genes RySto from the wild potato relative Solanum stoloniferum [21], the powdery mildew resistance gene Pm21 from the wild wheat relative Haynaldia villosa [79], and a species-wide inventory of NLR genes and alleles in Arabidopsis thaliana [71].

Two sequential polyploidization events followed by domestication and intensive breeding have narrowed the genetic diversity in cultivated wheat $[57,90]$. This dearth of diversity can be offset by introducing natural variation from wild species through wide crosses. In disease resistance breeding, resistance has been introgressed into wheat from at least 52 species from 13 genera due to the remarkable plasticity of the wheat genome [77]. Haynaldia villosa (genome constitution VV, $2 \mathrm{n}=14$ ) is a wild diploid species of wheat, which has been introduced into hexaploid bread wheat by the development of individual chromosome arm translocation lines between $H$. villosa and wheat. We previously reported the introgression from $H$. villosa into wheat of the powdery mildew resistance genes $P m 21, P m 55$ and $P m 62$, the yellow virus resistance gene $W s s 1$ and the cereal cyst nematode resistance gene $\mathrm{CreV}[14,16,83-85]$. Moreover, resistance to leaf rust, stripe rust, take-all, and sharp eye-spot resistances conferred by $H$. villosa chromatin in wheat have also been reported [26, 43, 52, 59]. The broad-spectrum resistance gene $P m 21$ has been widely used in wheat breeding and more than 40 new varieties have been 
released and cultivated in regions of China where powdery mildew is prevalent. Pm55 and Wss 1 have also been used in breeding programs and new wheat lines are currently under evaluation. Cloning of these resistance genes will accelerate their use by genetic engineering to reduce linkage drag. However, the lack of a reference genome and a high level of outcrossing of $H$. villosa makes resistance gene cloning in this species challenging.

Previously, we used SMRT-RenSeq to de novo assemble the NLRs of the inbred $H$. villosa accession 91C43. Then, NLR-Parser [67] was used to identify NLRs from the 1509 contigs, and 485 full-length NLRs were annotated [79]. In 2018, NLR-Annotator, the improved version for NLR prediction, was released (https://github. com/steuernb/NLR-Annotator). The improved software differs from NLR-Parser in that it can distinguish the border between different NLRs located in long contigs [69]. Since many NLRs are tightly clustered in plant genomes [46], we hypothesized that NLR-Annotator would identify a larger number of NLRs than previously predicted by NLR-Parser. Here NLR-Annotator was used to identify NLRs from $H$. villosa, followed by determination of the NLR classes, assignment of NLRs to chromosomes, exploration of NLRs orthologous to cloned R genes, identification of NLR-IDs and comparison of NLRs among different Triticeae species. Our study provides a valuable resource to support $R$ gene cloning in $H$. villosa and in $H$. villosa-wheat introgression lines.

\section{Results}

\section{NLR annotation in the SMRT-RenSeq assembly}

Previously, $406 \mathrm{Mb}$ of SMRT-RenSeq data consisting of 107,153 reads with an average length of $4.5 \mathrm{~kb}$ were generated and de novo assembled with Geneious v9.1.4 (Fig. S1). This assembly generated 1509 contigs in which $80 \%$ of the contigs ranged from $5 \mathrm{~kb}$ to $11 \mathrm{~kb}$, with the largest one spanning $24.6 \mathrm{~kb}$ (Fig. S2). The data was analyzed following the pipeline described in Fig. S3. In the present study, the 1509 contigs were re-analysed. Firstly, contigs sharing more than $95 \%$ identity were removed leaving 1456 non-redundant contigs. Secondly, sequences with low-complexity and with interspersed repeats were masked. Thirdly, and importantly, the contigs were annotated by NLR-Annotator, which, unlike our previous analysis, allows detection of multiple NLRs on the same contig. In total, 1320 NLRs were annotated in 1169 contigs, including 776 complete NLRs, 289 complete (pseudogene) NLRs, 188 partial NLRs and 67 partial (pseudogene) NLRs.

\section{Prediction and analysis of NLR domain composition}

To obtain the corresponding protein sequences of the annotated full-length NLRs, the genomic sequences of the identified NLRs were used to search the protein database of barley, wheat and Ae. tauschii using BLASTx. Then, the predicted $H$. villosa NLR protein sequences were obtained using FGENESH+ based on the homologous proteins. The 776 complete NLR protein candidates were analyzed by Plant_rgene to search for coiled-coil $(\mathrm{CC}), \mathrm{NB}-\mathrm{ARC}$ and integrated domains, then re-analyzed by InterProScan to search for the conserved LRR domains deposited in the SUPERFAMILY database. The results indicated that 772 members carried an NB-ARC, so these 772 candidates were considered to be bona fide NLRs. Then the 772 NLR proteins were further divided into five subclasses based on sub-domain analysis; 618 NLRs were typed as CC-NB-LRR (CNL), 98 as NB-LRR $(\mathrm{NL})$, three as CC-NB $(\mathrm{CN})$, one as NB-ARC $(\mathrm{N})$ and 52 as NLRs with integrated domains (NLR-ID) (Fig. 1).

The proteins were also downloaded from the newly released genomic database of several grass species, including T. aestivum, T. urartu, Ae. tauschii, $H$. vulgare, $O$. sativa and $B$. distachyon. Then, the NLRs in these species were identified using the same procedure as outlined above. The Triticeae $\mathrm{B}$ genome has the largest number of NLRs (834), followed by the V genome with 772 NLRs (Table 1). The Triticeae A, B, D, H and V genomes with an average of $632 \pm 148$ NLRs harbor more NLRs than $O$. sativa (347 NLRs) and B. distachyon ( $350 \mathrm{NLRs)}$ ), suggesting that Triticeae species have experienced NLR expansion more rapidly after diverging from $O$. sativa and $B$. distachyon. An NLR expansion in the A and D genome was also observed after polyploidization of wheat (Table 1).

The domain compositions of all the NLRs were analyzed by InterProScan and Plant_rgene. Unusually for $H$. villosa, $80 \%$ of the NLRs were of the CNL type compared to $\sim 50 \%$ in the other species. At first glance, this may suggest that different types of NLRs have traversed different evolutionary paths within the Triticeae. Alternatively, since the 'complete NLR' annotated by NLR-Annotator is defined as a sequence containing the P-loop, the start of the NB-ARC domain, as well as at least one LRR motif, the $\mathrm{CN}$ and $\mathrm{N}$ types NLRs were likely filtered out by our analysis.

\section{Phylogenetic analysis of NLRs in $\mathrm{H}$. villosa}

All 772 NLRs of $H$. villosa were used to construct a phylogenetic tree, based on the sequences of the NB-ARC domain, to reveal the potential evolutionary relationships (Fig. 2, https://itol.embl.de/shared/2018201031). The conserved motifs from each complete NLR were displayed in the phylogenetic tree to track the evolutionary characteristics of each clade; 11 main clades were thus derived and displayed in different colors. The structure and characteristics of each clade was analyzed according 


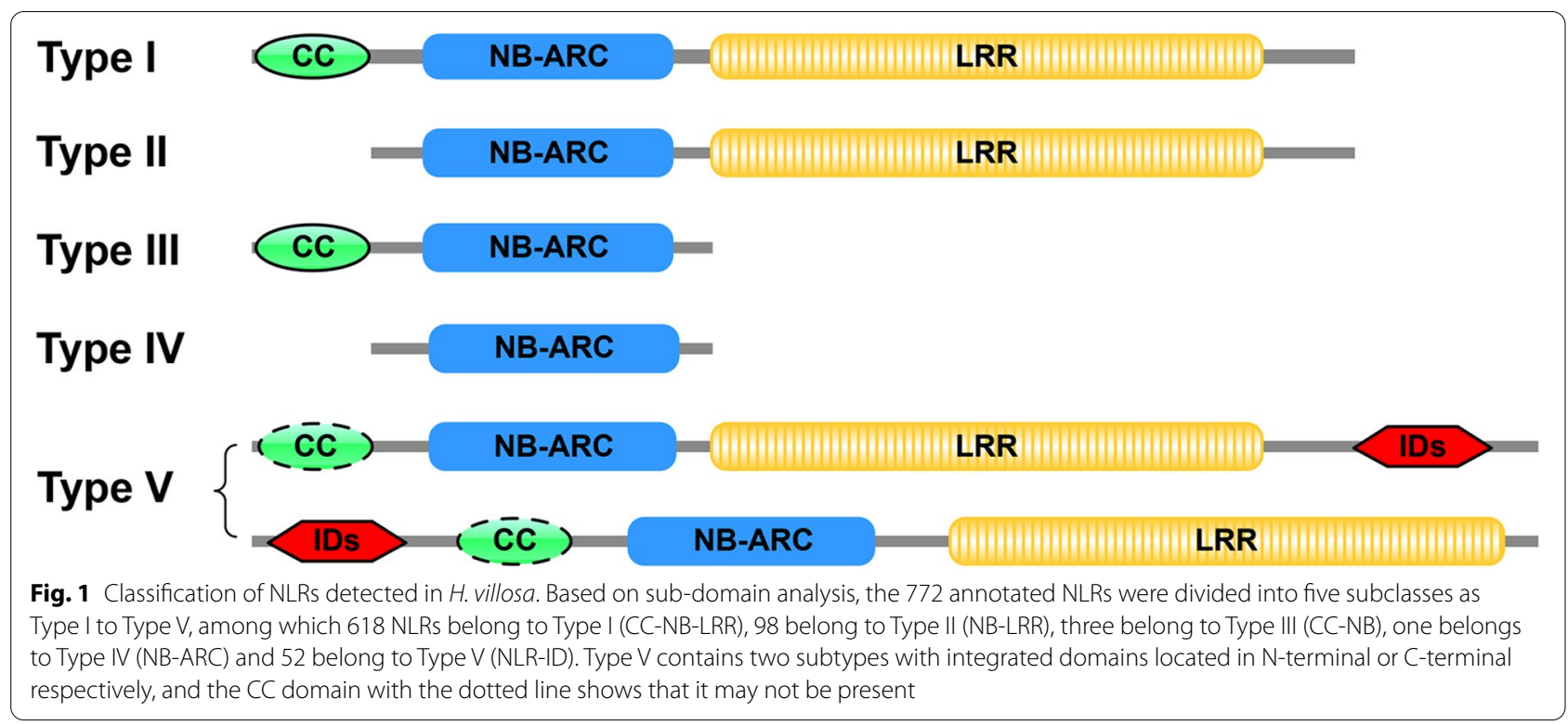

Table 1 NLRs identified in H. villosa and evolutionary related species

\begin{tabular}{|c|c|c|c|c|c|c|c|c|c|c|c|}
\hline \multirow[t]{2}{*}{ Species } & \multirow[t]{2}{*}{ Total NLR genes } & \multicolumn{2}{|l|}{ CNL } & \multicolumn{2}{|l|}{ NL } & \multicolumn{2}{|l|}{$\mathrm{CN}$} & \multicolumn{2}{|l|}{$\mathbf{N}$} & \multicolumn{2}{|c|}{ NLR-ID } \\
\hline & & Num. & Pct. & Num. & Pct. & Num. & Pct. & Num. & Pct. & Num. & Pct. \\
\hline B. distachyon & 350 & 201 & $57.4 \%$ & 60 & $17.1 \%$ & 53 & $15.1 \%$ & 18 & $5.1 \%$ & 16 & $4.6 \%$ \\
\hline O. sativa & 347 & 156 & $45.0 \%$ & 70 & $20.2 \%$ & 77 & $22.2 \%$ & 35 & $10.1 \%$ & 7 & $2.0 \%$ \\
\hline H. vulgare & 397 & 198 & $50.0 \%$ & 84 & $21.2 \%$ & 60 & $15.1 \%$ & 31 & $7.8 \%$ & 24 & $6.1 \%$ \\
\hline T. urartu & 530 & 275 & $52.0 \%$ & 107 & $20.2 \%$ & 78 & $14.7 \%$ & 24 & $4.5 \%$ & 45 & $8.5 \%$ \\
\hline A. tauschii & 572 & 298 & $52.1 \%$ & 113 & $19.8 \%$ & 70 & $12.2 \%$ & 50 & $8.7 \%$ & 41 & $7.2 \%$ \\
\hline T. aestivum & 2273 & 1181 & $52.0 \%$ & 367 & $16.2 \%$ & 493 & $21.7 \%$ & 98 & $4.3 \%$ & 130 & $5.7 \%$ \\
\hline (A genome) & $(672)$ & 346 & $51.5 \%$ & 102 & $15.2 \%$ & 153 & $22.8 \%$ & 32 & $4.8 \%$ & 38 & $5.7 \%$ \\
\hline (B genome) & (834) & 443 & $53.1 \%$ & 146 & $17.5 \%$ & 169 & $20.3 \%$ & 27 & $3.2 \%$ & 47 & $5.7 \%$ \\
\hline (D genome) & (647) & 339 & $52.4 \%$ & 100 & $15.5 \%$ & 135 & $20.9 \%$ & 34 & $5.7 \%$ & 38 & $5.9 \%$ \\
\hline H. villosa & 772 & 618 & $80.1 \%$ & 98 & $12.7 \%$ & 3 & $0.39 \%$ & 1 & $0.13 \%$ & 52 & $6.8 \%$ \\
\hline
\end{tabular}

Note: Num. number, Pct. percentage (\%)

to the motif classification by Jupe [33]. Clade A is composed of NLs lacking CC domains. Clade B contains NLR-IDs carrying exclusively the DDE_Tnp_4 domain. Clade C members lack the NB-ARC 'motif 10', and the CC 'motif 17 ' is followed by 'motif 15 ' but not by 'motif 16 ' as is usually the case. 'Motif 15, also referred to as 'TIR-2, is found in both monocots and dicots [33]. In this study the annotated NLRs containing this motif were still classified as being CNL but not TNL due to the CC domain being detected. All the NLRs in clade $\mathrm{C}$ are located on chromosome $3 \mathrm{~V}$, indicating that this type of NLR has experienced active expansion but not migration. Clade $\mathrm{D}$ members contain only 'motif 16 ' in the $\mathrm{CC}$ domain but lack 'motif 17'. Clade E NB-ARC domains contain an additional 'motif 6 ' followed by 'motif 1'. Clade H members contain a longer linker between the $\mathrm{CC}$ domain and the NB-ARC domain, and the LRR domain is more irregular. Clade I contains three or four tandem repeats

(See figure on next page.)

Fig. 2 Phylogenetic analysis of 772 NLRs based on the NB-ARC domain. The phylogenetic tree of the 772 NLR of H. villosa was constructed based on the sequences of the NB-ARC domain using MEGA7 by Neighbor-Joining method with 1000 bootstrap replicates, and the tree was visualized using iTOL (https://itol.embl.de/shared/2018201031). Eleven main clades were displayed using different colors in the tree, and the conserved motifs from each complete NLR were displayed using different colors in the domain compositions. Number 1 to 7 present in silico localization of NLR genes on chromosome $1 \mathrm{~V}$ to $7 \mathrm{~V}$ 


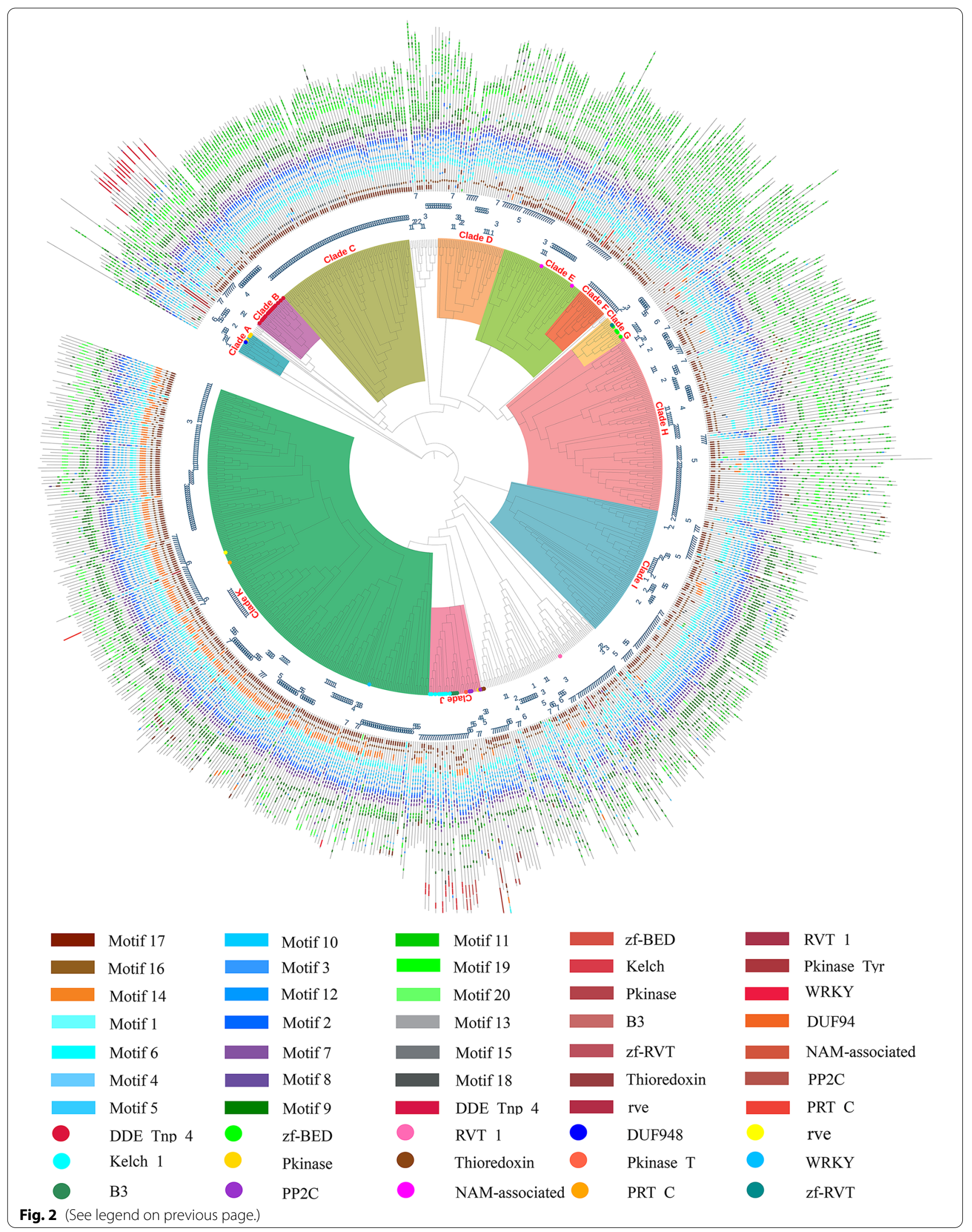


of 'motif 9' following the linker. Clade F lacks 'motif 10' in the NB-ARC domain. Clade $G$ and J members tend to contain one of either three or five, respectively, integrated domains. Clade $\mathrm{K}$ is mainly composed of CNLs and a few NLs. In this clade, most members contain 'motif 14 ' between the $\mathrm{CC}$ and NB-ARC domains, a signature reported to be unique in monocots [33]. Another characteristic of clade $\mathrm{K}$ is that eight motifs associated with the NB-ARC domain are arranged in a uniform order. Compared to the CC and NB-ARC domains, a larger diversity was found in the LRR domain, such as motif numbers and arrangements, possibly reflecting the role of the LRRs in recognizing pathogen effectors.

\section{NLR expression analysis}

We sequenced a full-length transcriptome of $H$. villosa with PacBio SMRT chemistry. To detect expressed NLRs and to verify the predicted exon/intron boundaries, the 772 NLRs were searched against NLRs annotated in the transcriptome database. This revealed 91 NLRs with a transcript with more than $>95 \%$ identity and a query coverage $>90 \%$. Sequence alignment between the transcript and the genomic DNA of these 91 NLRs indicated that the splicing pattern of 85 of the NLRs perfectly matched our predictions. The remaining 6 expressed $N L R s$, whose splicing patterns didn't match with the predictions, were used to test whether specific splicing patterns happened in $H$. villosa by comparing their splicing patterns with those of the corresponding orthologous NLRs. The comparisons indicated that there were no specific splicing patterns happened in 3 NLRs, including Hv_Contig_1470_nlr_1, Hv_Contig_696_nlr_1 and Hv_ Contig_1466_nlr_1, while the coding lengths were different between the predicted sequences and the expressed sequences. For Hv_Contig_249_nlr_2, its orthologous NLRs in wheat barley and Ae. tauschii contained A and B types of splicing patterns, and the previously predicted CDS was corresponding to the A type, while the expressed CDS was corresponding to the B type. So, there was no specific splicing pattern in Hv_Contig_249_nlr_2. However, the specific splicing patterns happened in $\mathrm{Hv}_{-}$ Contig_670_nlr_1 and Hv_Contig_61_nlr_1. Knowledge of NLR expression can provide additional powerful support for $R$ gene cloning.

\section{Assignment of NLRs to chromosomes}

$H$. villosa displays good chromosome collinearity with barley, Ae. tauchii, Triticum urartu and wheat. Therefore the nucleotide sequences of the 772 complete NLRs were used as a query for BLASTn analysis against the genome databases of these species for in silico prediction of chromosomal location. This procedure assigned all 772 complete NLRs to homoelogous chromosomes; 139 NLRs to group 1, 85 to group 2, 164 to group 3, 15 to group 4, 60 to group 5, 101 to group 6, and 208 to group 7 (Table S3). However, due to the differentiation between the $H$. villosa genome and those of the species used in the analysis, we decided to independently confirm the assignment for a subset of NLRs. To this end, we took advantage of a previously developed full set of wheat- $H$. villosa translocation lines, each involving one of the 14 chromosome arms of $H$. villosa [86], to physically locate NLRs using PCR molecular markers. A subset of the in silico mapped NLRs were selected evenly from the seven chromosomes. The sequence of each selected $N L R$ was aligned with the predicted wheat orthologues on the A, B, and D genome, then primers were designed based on insertions or deletions private to $H$. villosa. A total of 757 primer pairs were designed corresponding to 565 contigs, of which 105 primer pairs gave rise to polymorphisms between $H$. villosa and wheat. Thus, the polymorphism rate produced using InDel-markers based on NLRs was 14\%, which is significantly lower than the $52 \%$ produced using IT (Intron-Target)-markers based on single copy genes [87]. The high sequence similarity between NLRs likely complicates the design of polymorphic markers.

From the 105 polymorphic primer pairs, 61 complete and 26 partial (pseudogene) NLRs could be located to specific chromosome arms (Fig. 3; Table 2). All the NLRs located in silico on homoeologous groups 1 , $2,3,5$ and 6 were physically located on chromosome $1 \mathrm{~V}, 2 \mathrm{~V}, 3 \mathrm{~V}, 5 \mathrm{~V}$ and $6 \mathrm{~V}$, respectively, thus showing a perfect homoeologous relationship between groups $1,2,3,5$ and 6 , respectively, of $H$. villosa and other Triticum species (Table 2). As to the distribution of NLRs on $4 \mathrm{~V}$, the NLRs physically located on $4 \mathrm{VS}$ were located in silico on homoeologous groups 4 , however, NLRs physically located on 4VL were largely in silico predicted to be located on the homoeologous group 7 (Table 2). It was also previously reported that four 4VL-specific markers of $H$. villosa detected homoeoloci on the group 7 chromosomes of wheat [86]. Therefore, our data, and those of Zhang et al. [86], suggest that $4 \mathrm{VL}$ did not translocate with $7 \mathrm{VS}$, unlike the reciprocal $4 \mathrm{~L} / 7 \mathrm{~S}$ translocation which happened in wheat and other Triticeae species.

\section{Enrichment efficiency of NLR loci corresponding to cloned R genes}

In the TSLMMHV1 bait design, baits corresponding to all the cloned barley Mla genes were added manually. To test the efficiency of enrichment, the orthologous $H$. villosa Mla genes were obtained from our $H$. villosa NLR assembly. In total, 18 NLRs in $H$. villosa were predicted to be orthologous to Mla genes because they showed the highest homology to NLRs at the barley Mla locus 

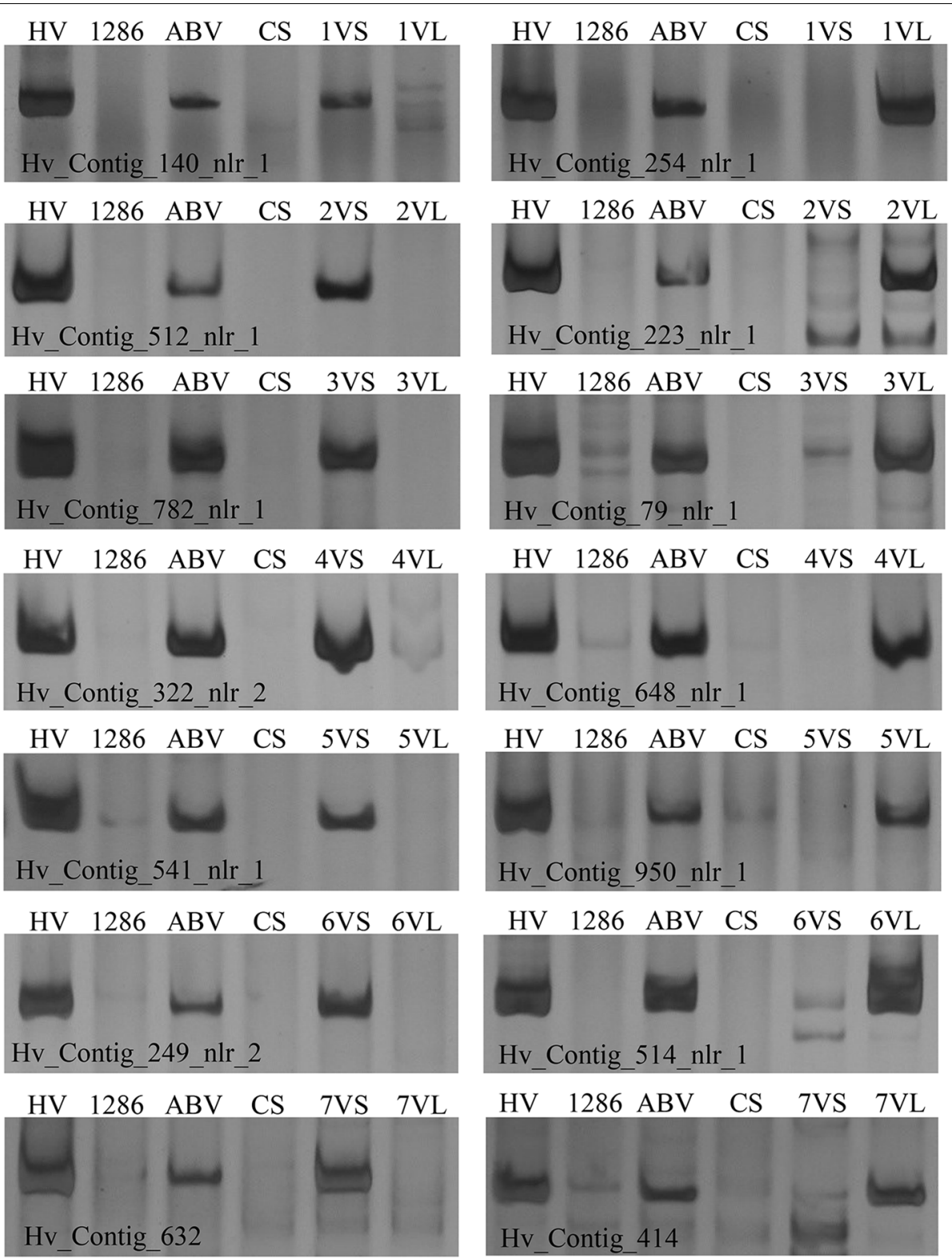

Fig. 3 Chromosomal location of the annotated NLRs using a full set of 14 whole arm translocation lines. HV: H. villosa $(2 n=14, W)$; $Z Y 1286: T$. turgidum tetraploid wheat $(2 n=28, A A B B)$; ABV: T. turgidum-H. villosa hexaploid amphiploid wheat $(2 n=42, A A B B V)$; CS: hexaploid wheat Chinese Spring $(2 \mathrm{n}=42$, AABBDD), 1VS, 2VS, 3VS, 4VS, 5VS, 6VS, 7VS: wheat-H. villosa translocation lines involving the short arm of 1V, 2V, 3V, 4V, 5V, 6V, 7V; $1 \mathrm{VL}, 2 \mathrm{VL}, 3 \mathrm{VL}, 4 \mathrm{VL}, 5 \mathrm{VL}, 6 \mathrm{VL}, 7 \mathrm{VL}$ : wheat-H. villosa translocation lines involved the long arm of $1 \mathrm{~V}, 2 \mathrm{~V}, 3 \mathrm{~V}, 4 \mathrm{~V}, 5 \mathrm{~V}, 6 \mathrm{~V}, 7 \mathrm{~V}$

at $30.2 \mathrm{Mb}$ on chromosome $1 \mathrm{H}$ (Table 3 ). When using the rye $\mathrm{Sr} 50$, the orthologous gene of barley Mla, as the query, the same set of homologous genes were obtained. It was reported that the number of Mla paralogues was five in wheat and barley but expanded to over 20 in rye [49]. We detected 19 paralogues in $H$. villosa indicating that the expansion of Mla genes also occurred in $\mathrm{H}$. villosa. In barley, Mla paralogues also occur at a second locus at $\sim 8.6 \mathrm{Mb}$ on chromosome $1 \mathrm{H}$; we obtained 15
Mla paralogues in $H$. villosa corresponding to this locus. The orthologous genes of Mla in other species confer resistance to different diseases, such as powdery mildew by TmMLA1 from Triticum monococcum [31], and stem rust by $\mathrm{Sr} 50$ from Secale cereale and Sr33 from Aegilops tauschii $[49,56]$. Moreover, barley Mla3, which confers resistance to barley powdery mildew [1], has recently been shown to also confer resistance to rice blast [10]. Our data suggest that the number of $M L A$ paralogues 
Table 2 Chromosomal location of the identified NLRs in H. villosa

\begin{tabular}{|c|c|c|c|c|c|}
\hline \multirow[t]{2}{*}{ NLR genes } & \multirow[t]{2}{*}{ NLR status } & \multicolumn{4}{|c|}{ Chromosomal Location } \\
\hline & & Triticum aestivum & Hordeum vulgare & Aegilops tauschii & $\begin{array}{l}\text { Haynaldia } \\
\text { villosa }\end{array}$ \\
\hline Hv_Contig_60_nlr_3 & Complete NLR & $1 \mathrm{D}$ & $1 \mathrm{H}$ & $1 \mathrm{D}$ & $1 \mathrm{VS}$ \\
\hline Hv_Contig_138_nlr_2 & Complete NLR & $1 \mathrm{~A}$ & $1 \mathrm{H}$ & $1 \mathrm{D}$ & $1 \mathrm{VS}$ \\
\hline Hv_Contig_140_nlr_1 & Complete NLR & $1 \mathrm{D}$ & $1 \mathrm{H}$ & $1 \mathrm{D}$ & $1 \mathrm{VS}$ \\
\hline Hv_Contig_232_nlr_1 & Complete NLR & $1 \mathrm{~B}$ & $1 \mathrm{H}$ & $1 \mathrm{D}$ & $1 \mathrm{VS}$ \\
\hline Hv_Contig_443_nlr_1 & Complete NLR & $1 \mathrm{~B}$ & $1 \mathrm{H}$ & $1 \mathrm{D}$ & $1 \mathrm{VS}$ \\
\hline Hv_Contig_992_nlr_1 & Complete NLR & $1 \mathrm{~A}$ & $1 \mathrm{H}$ & $1 \mathrm{D}$ & $1 \mathrm{VS}$ \\
\hline Hv_Contig_1391_nlr_1 & Complete NLR & $1 \mathrm{~A}$ & $1 \mathrm{H}$ & $1 \mathrm{D}$ & $1 \mathrm{VS}$ \\
\hline Hv_Contig_254_nlr_1 & Complete NLR & $1 \mathrm{~A}$ & $1 \mathrm{H}$ & $1 \mathrm{D}$ & $1 \mathrm{VL}$ \\
\hline Hv_Contig_452_nlr_1 & Complete NLR & $1 \mathrm{D}$ & $1 \mathrm{H}$ & $1 \mathrm{D}$ & $1 \mathrm{VL}$ \\
\hline Hv_Contig_1453_nlr_1 & Complete NLR & $1 \mathrm{D}$ & $1 \mathrm{H}$ & $1 \mathrm{D}$ & $1 \mathrm{VL}$ \\
\hline Hv_Contig_951 & Partial or pseudogene & $1 \mathrm{~A}$ & $3 \mathrm{H}$ & $1 \mathrm{D}$ & $1 \mathrm{VS}$ \\
\hline Hv_Contig_1193 & Partial or pseudogene & $1 \mathrm{D}$ & $1 \mathrm{H}$ & $1 \mathrm{D}$ & $1 \mathrm{VS}$ \\
\hline Hv_Contig_141 & Partial or pseudogene & $1 \mathrm{~A}$ & $1 \mathrm{H}$ & $1 \mathrm{D}$ & $1 \mathrm{VL}$ \\
\hline Hv_Contig_219 & Partial or pseudogene & $1 \mathrm{D}$ & $1 \mathrm{H}$ & $1 \mathrm{D}$ & $1 \mathrm{VL}$ \\
\hline Hv_Contig_512_nlr_1 & Complete NLR & $2 \mathrm{~A}$ & $2 \mathrm{H}$ & $2 \mathrm{D}$ & $2 \mathrm{VS}$ \\
\hline Hv_Contig_544_nlr_2 & Complete NLR & $2 \mathrm{D}$ & $2 \mathrm{H}$ & $2 \mathrm{D}$ & $2 \mathrm{VS}$ \\
\hline Hv_Contig_223_nlr_1 & Complete NLR & $2 \mathrm{~B}$ & $2 \mathrm{H}$ & $2 \mathrm{D}$ & $2 \mathrm{VL}$ \\
\hline Hv_Contig_1028_nlr_1 & Complete NLR & $2 \mathrm{D}$ & $2 \mathrm{H}$ & $2 \mathrm{D}$ & $2 \mathrm{VL}$ \\
\hline Hv_Contig_461_nlr_1 & Complete NLR & $2 \mathrm{D}$ & $2 \mathrm{H}$ & $2 \mathrm{D}$ & $2 \mathrm{VL}$ \\
\hline Hv_Contig_1254 & Partial or pseudogene & Un & $2 \mathrm{H}$ & $2 \mathrm{D}$ & $2 \mathrm{VS}$ \\
\hline Hv_Contig_35 & Partial or pseudogene & $2 \mathrm{D}$ & $2 \mathrm{H}$ & $2 \mathrm{D}$ & $2 \mathrm{VL}$ \\
\hline Hv_Contig_139 & Partial or pseudogene & $2 \mathrm{D}$ & $2 \mathrm{H}$ & $2 \mathrm{D}$ & $2 \mathrm{VL}$ \\
\hline Hv_Contig_667 & Partial or pseudogene & $2 \mathrm{~B}$ & $2 \mathrm{H}$ & $2 \mathrm{D}$ & $2 \mathrm{VL}$ \\
\hline Hv_Contig_716_nlr_1 & Complete NLR & $3 \mathrm{~A}$ & $3 \mathrm{H}$ & $3 D$ & $3 \mathrm{VS}$ \\
\hline Hv_Contig_782_nlr_1 & Complete NLR & $3 B$ & $3 \mathrm{H}$ & $3 D$ & $3 \mathrm{VS}$ \\
\hline Hv_Contig_11_nlr_1 & Complete NLR & $3 \mathrm{D}$ & $3 \mathrm{H}$ & $3 D$ & $3 \mathrm{VL}$ \\
\hline Hv_Contig_79_nlr_1 & Complete NLR & $3 B$ & $3 \mathrm{H}$ & $3 D$ & $3 \mathrm{VL}$ \\
\hline Hv_Contig_326_nlr_1 & Complete NLR & $3 \mathrm{D}$ & $3 \mathrm{H}$ & $3 D$ & $3 \mathrm{VL}$ \\
\hline Hv_Contig_657_nlr_2 & Complete NLR & $3 \mathrm{~A}$ & $3 \mathrm{H}$ & $3 D$ & $3 V L$ \\
\hline Hv_Contig_866_nlr_1 & Complete NLR & $3 B$ & $3 \mathrm{H}$ & $3 D$ & $3 \mathrm{VL}$ \\
\hline Hv_Contig_77 & Partial or pseudogene & $3 \mathrm{~A}$ & $3 \mathrm{H}$ & $3 D$ & $3 \mathrm{VL}$ \\
\hline Hv_Contig_686 & Partial or pseudogene & $3 B$ & $3 \mathrm{H}$ & $3 D$ & $3 \mathrm{VL}$ \\
\hline Hv_Contig_90_nlr_1 & Complete NLR & $4 \mathrm{~B}$ & Un & $4 \mathrm{D}$ & $4 \mathrm{VS}$ \\
\hline Hv_Contig_116_nlr_1 & Complete NLR & $4 \mathrm{D}$ & $4 \mathrm{H}$ & $4 \mathrm{D}$ & 4VS \\
\hline Hv_Contig_322_nlr_2 & Complete NLR & $4 \mathrm{~B}$ & $4 \mathrm{H}$ & $4 \mathrm{D}$ & 4VS \\
\hline Hv_Contig_670_nlr_1 & Complete NLR & $4 \mathrm{~B}$ & $4 \mathrm{H}$ & $4 \mathrm{D}$ & 4VS \\
\hline Hv_Contig_28_nlr_1 & Complete NLR & $7 \mathrm{~B}$ & $7 \mathrm{H}$ & $7 D$ & $4 \mathrm{VL}$ \\
\hline Hv_Contig_55_nlr_1 & Complete NLR & $7 D$ & $7 \mathrm{H}$ & $7 D$ & $4 \mathrm{VL}$ \\
\hline Hv_Contig_82_nlr_2 & Complete NLR & $7 D$ & $7 \mathrm{H}$ & $7 D$ & $4 \mathrm{VL}$ \\
\hline Hv_Contig_172_nlr_1 & Complete NLR & $7 B$ & $7 \mathrm{H}$ & $7 D$ & $4 \mathrm{VL}$ \\
\hline Hv_Contig_299_nlr_1 & Complete NLR & $7 D$ & $7 \mathrm{H}$ & $7 D$ & $4 \mathrm{VL}$ \\
\hline Hv_Contig_393_nlr_1 & Complete NLR & $7 \mathrm{~A}$ & $7 \mathrm{H}$ & $7 D$ & $4 \mathrm{VL}$ \\
\hline Hv_Contig_648_nlr_1 & Complete NLR & $7 D$ & $7 \mathrm{H}$ & $7 D$ & $4 \mathrm{VL}$ \\
\hline Hv_Contig_798_nlr_1 & Complete NLR & $7 D$ & $7 \mathrm{H}$ & $7 D$ & $4 \mathrm{VL}$ \\
\hline Hv_Contig_913_nlr_2 & Complete NLR & $7 D$ & $7 \mathrm{H}$ & $7 D$ & $4 \mathrm{VL}$ \\
\hline Hv_Contig_958_nlr_1 & Complete NLR & $7 B$ & $7 \mathrm{H}$ & $7 D$ & $4 \mathrm{VL}$ \\
\hline Hv_Contig_1239_nlr_1 & Complete NLR & $7 \mathrm{~A}$ & $7 \mathrm{H}$ & $7 D$ & $4 \mathrm{VL}$ \\
\hline
\end{tabular}


Table 2 (continued)

\begin{tabular}{|c|c|c|c|c|c|}
\hline \multirow[t]{2}{*}{ NLR genes } & \multirow[t]{2}{*}{ NLR status } & \multicolumn{4}{|c|}{ Chromosomal Location } \\
\hline & & Triticum aestivum & Hordeum vulgare & Aegilops tauschii & $\begin{array}{l}\text { Haynaldia } \\
\text { villosa }\end{array}$ \\
\hline Hv_Contig_1300_nlr_1 & Complete NLR & $7 D$ & $7 \mathrm{H}$ & $7 D$ & $4 \mathrm{VL}$ \\
\hline Hv_Contig_1318_nlr_1 & Complete NLR & $7 D$ & $7 \mathrm{H}$ & $7 D$ & $4 \mathrm{VL}$ \\
\hline Hv_Contig_1410_nlr_1 & Complete NLR & $7 D$ & $7 \mathrm{H}$ & $7 D$ & $4 \mathrm{VL}$ \\
\hline Hv_Contig_120 & Partial or pseudogene & $4 \mathrm{~B}$ & $4 \mathrm{H}$ & $4 \mathrm{D}$ & 4VS \\
\hline Hv_Contig_362 & Partial or pseudogene & $4 \mathrm{~A}$ & $7 \mathrm{H}$ & $7 D$ & $4 \mathrm{VL}$ \\
\hline Hv_Contig_1235 & Partial or pseudogene & $7 \mathrm{~A}$ & $2 \mathrm{H}$ & $7 D$ & $4 \mathrm{VL}$ \\
\hline Hv_Contig_1339 & Partial or pseudogene & $4 \mathrm{~A}$ & $7 \mathrm{H}$ & $7 D$ & $4 \mathrm{VL}$ \\
\hline Hv_Contig_541_nlr_1 & Complete NLR & $5 \mathrm{D}$ & $5 \mathrm{H}$ & $5 \mathrm{D}$ & $5 \mathrm{VS}$ \\
\hline Hv_Contig_1146_nlr_1 & Complete NLR & $7 \mathrm{D}$ & $5 \mathrm{H}$ & $7 \mathrm{D}$ & $5 \mathrm{VS}$ \\
\hline Hv_Contig_105_nlr_1 & Complete NLR & $5 \mathrm{D}$ & $5 \mathrm{H}$ & $5 \mathrm{D}$ & $5 \mathrm{VL}$ \\
\hline Hv_Contig_253_nlr_1 & Complete NLR & $5 \mathrm{~A}$ & $5 \mathrm{H}$ & $5 D$ & $5 \mathrm{VL}$ \\
\hline Hv_Contig_308_nlr_1 & Complete NLR & $5 B$ & $5 \mathrm{H}$ & $7 D$ & $5 \mathrm{VL}$ \\
\hline Hv_Contig_705_nlr_1 & Complete NLR & $5 \mathrm{D}$ & $5 \mathrm{H}$ & $5 \mathrm{D}$ & $5 \mathrm{VL}$ \\
\hline Hv_Contig_757_nlr_1 & Complete NLR & $5 \mathrm{D}$ & $5 \mathrm{H}$ & $5 \mathrm{D}$ & $5 \mathrm{VL}$ \\
\hline Hv_Contig_937_nlr_1 & Complete NLR & $5 B$ & Un & $5 D$ & $5 \mathrm{VL}$ \\
\hline Hv_Contig_950_nlr_1 & Complete NLR & $5 B$ & $5 \mathrm{H}$ & $5 \mathrm{D}$ & $5 \mathrm{VL}$ \\
\hline Hv_Contig_85 & Partial or pseudogene & $5 B$ & $5 \mathrm{H}$ & $2 \mathrm{D}$ & $5 \mathrm{VS}$ \\
\hline Hv_Contig_270 & Partial or pseudogene & $5 B$ & $5 \mathrm{H}$ & $5 \mathrm{D}$ & $5 \mathrm{VL}$ \\
\hline Hv_Contig_1353 & Partial or pseudogene & $5 \mathrm{~A}$ & $5 \mathrm{H}$ & $6 \mathrm{D}$ & $5 \mathrm{VL}$ \\
\hline Hv_Contig_665_nlr_1 & Complete NLR & $6 \mathrm{~B}$ & $6 \mathrm{H}$ & $6 \mathrm{D}$ & $6 \mathrm{VS}$ \\
\hline Hv_Contig_39_nlr_2 & Complete NLR & $6 \mathrm{~B}$ & $6 \mathrm{H}$ & $6 \mathrm{D}$ & 6VS \\
\hline Hv_Contig_249_nlr_2 & Complete NLR & $6 \mathrm{~B}$ & $6 \mathrm{H}$ & $6 \mathrm{D}$ & $6 \mathrm{VS}$ \\
\hline Hv_Contig_514_nlr_1 & Complete NLR & $6 \mathrm{D}$ & $6 \mathrm{H}$ & $6 \mathrm{D}$ & $6 \mathrm{VL}$ \\
\hline Hv_Contig_750_nlr_1 & Complete NLR & $6 \mathrm{~B}$ & $6 \mathrm{H}$ & $5 \mathrm{D}$ & $6 \mathrm{VL}$ \\
\hline Hv_Contig_860_nlr_1 & Complete NLR & $6 \mathrm{~A}$ & $6 \mathrm{H}$ & $6 \mathrm{D}$ & $6 \mathrm{VL}$ \\
\hline Hv_Contig_1012 & Partial or pseudogene & $6 \mathrm{~A}$ & $6 \mathrm{H}$ & $6 \mathrm{D}$ & 6VS \\
\hline Hv_Contig_1162 & Partial or pseudogene & $6 \mathrm{~B}$ & $6 \mathrm{H}$ & Un & 6VS \\
\hline Hv_Contig_736 & Partial or pseudogene & $6 \mathrm{D}$ & Un & $6 \mathrm{D}$ & 6VS \\
\hline Hv_Contig_539 & Partial or pseudogene & $6 \mathrm{~B}$ & $6 \mathrm{H}$ & $6 \mathrm{D}$ & $6 \mathrm{VL}$ \\
\hline Hv_Contig_38_nlr_2 & Complete NLR & $7 \mathrm{~A}$ & $7 \mathrm{H}$ & $7 \mathrm{D}$ & 7VS \\
\hline Hv_Contig_55_nlr_1 & Complete NLR & $7 D$ & $7 \mathrm{H}$ & $7 \mathrm{D}$ & 7VS \\
\hline Hv_Contig_99_nlr_3 & Complete NLR & $7 D$ & $7 \mathrm{H}$ & $7 D$ & $7 \mathrm{VL}$ \\
\hline Hv_Contig_508_nlr_1 & Complete NLR & $4 \mathrm{~A}$ & $7 \mathrm{H}$ & $3 \mathrm{D}$ & $7 \mathrm{VL}$ \\
\hline Hv_Contig_1010_nlr_1 & Complete NLR & $7 \mathrm{~B}$ & Un & $7 \mathrm{D}$ & $7 \mathrm{VL}$ \\
\hline Hv_Contig_1236_nlr_1 & Complete NLR & $7 D$ & $7 \mathrm{H}$ & $7 D$ & $7 \mathrm{VL}$ \\
\hline Hv_Contig_632 & Partial or pseudogene & $7 \mathrm{~B}$ & $7 \mathrm{H}$ & $7 D$ & 7VS \\
\hline Hv_Contig_912 & Partial or pseudogene & $7 \mathrm{~A}$ & $7 \mathrm{H}$ & $7 D$ & 7VS \\
\hline Hv_Contig_414 & Partial or pseudogene & $4 \mathrm{~A}$ & $7 \mathrm{H}$ & $7 \mathrm{D}$ & $7 \mathrm{VL}$ \\
\hline Hv_Contig_940 & Partial or pseudogene & $7 D$ & $7 \mathrm{H}$ & $7 \mathrm{D}$ & $7 \mathrm{VL}$ \\
\hline Hv_Contig_978 & Partial or pseudogene & $7 \mathrm{~A}$ & $4 \mathrm{H}$ & $7 D$ & $7 \mathrm{VL}$ \\
\hline
\end{tabular}

Note: Un indicates that the chromosomal location of the matched gene was unknown

expanded at both sites in $H$. villosa providing rich candidates to mine new resistance genes.

We used several other cloned NLR genes to test the enrichment efficiency. Six NLRs in silico-located on $1 \mathrm{~V}$ showed highest homology to the Pm3 locus and as such were predicted to be H. villosa Pm3 orthologues. Similarly, orthologues for $S r 45, Y r 7, R C R 1, S r 35, L r 1$, and $P m 2$ could also be successfully identified in $H$. villosa from $1 \mathrm{~V}$, $2 \mathrm{~V}, 3 \mathrm{~V}$ and $5 \mathrm{~V}$ respectively. No orthologues of wheat $T s n-1, S r 22$ or $L r 10$ were detected on the corresponding 
Table 3 Orthologous genes in H. villosa corresponding to the reported NLR genes

\begin{tabular}{|c|c|c|c|c|c|c|}
\hline & Contig number & $\begin{array}{l}\text { In silico } \\
\text { location }\end{array}$ & Type of NLR & $\begin{array}{l}\text { Length of protein } \\
\text { (aa) }\end{array}$ & $\begin{array}{l}\text { Identity to } \\
\text { reference (\%) }\end{array}$ & $\begin{array}{l}\text { Coverage } \\
\text { (\%) }\end{array}$ \\
\hline \multirow{18}{*}{$\begin{array}{l}\text { Mla1 and Sr50 } \\
\text { (at 30.2 Mb) }\end{array}$} & Hv_Contig_1079_nlr_1 & $1 \mathrm{~V}$ & $\mathrm{CNL}$ & 967 & 86.7 & 86.0 \\
\hline & Hv_Contig_343_nlr_1 & $1 \mathrm{~V}$ & $\mathrm{CNL}$ & 960 & 86.6 & 86.3 \\
\hline & Hv_Contig_1392_nlr_1 & $1 \mathrm{~V}$ & $\mathrm{CNL}$ & 955 & 86.9 & 86.0 \\
\hline & Hv_Contig_1173_nlr_1 & $1 \mathrm{~V}$ & $\mathrm{CNL}$ & 949 & 86.0 & 88.2 \\
\hline & Hv_Contig_1386_nlr_1 & $1 \mathrm{~V}$ & $\mathrm{CNL}$ & 954 & 85.4 & 84.2 \\
\hline & Hv_Contig_857_nlr_1 & $1 \mathrm{~V}$ & $\mathrm{CNL}$ & 973 & 85.0 & 84.1 \\
\hline & Hv_Contig_60_nlr_2 & $1 \mathrm{~V}$ & $\mathrm{CNL}$ & 866 & 87.8 & 87.9 \\
\hline & Hv_Contig_212_nlr_1 & $1 \mathrm{~V}$ & $\mathrm{CNL}$ & 951 & 87.9 & 86.2 \\
\hline & Hv_Contig_115_nlr_1 & $1 \mathrm{~V}$ & $\mathrm{CNL}$ & 941 & 87.4 & 83.5 \\
\hline & Hv_Contig_653_nlr_1 & $1 \mathrm{~V}$ & $\mathrm{CNL}$ & 882 & 87.5 & 85.9 \\
\hline & Hv_Contig_1211_nlr_1 & $1 \mathrm{~V}$ & $\mathrm{CNL}$ & 880 & 86.4 & 79.3 \\
\hline & Hv_Contig_605_nlr_1 & $1 \mathrm{~V}$ & $\mathrm{CNL}$ & 886 & 87.5 & 79.8 \\
\hline & Hv_Contig_181_nlr_1 & $1 \mathrm{~V}$ & $\mathrm{CNL}$ & 967 & 86.6 & 88.0 \\
\hline & Hv_Contig_1213_nlr_1 & $1 \mathrm{~V}$ & $\mathrm{CNL}$ & 895 & 85.1 & 86.4 \\
\hline & Hv_Contig_436_nlr_1 & $1 \mathrm{~V}$ & $\mathrm{CNL}$ & 966 & 85.9 & 84.7 \\
\hline & Hv_Contig_188_nlr_1 & $1 \mathrm{~V}$ & $\mathrm{CNL}$ & 913 & 85.8 & 80.4 \\
\hline & Hv_Contig_298_nlr_1 & $1 \mathrm{~V}$ & $\mathrm{CNL}$ & 955 & 84.7 & 85.9 \\
\hline & Hv_Contig_650_nlr_1 & $1 \mathrm{~V}$ & $\mathrm{CNL}$ & 883 & 84.8 & 86.0 \\
\hline \multirow{15}{*}{$\begin{array}{l}\text { Mla1 and Sr50 } \\
\text { (at } \sim 8.6 \mathrm{Mb})\end{array}$} & Hv_Contig_751_nlr_1 & $1 \mathrm{~V}$ & $\mathrm{CNL}$ & 921 & 84.7 & 78.6 \\
\hline & Hv_Contig_1287_nlr_1 & $1 \mathrm{~V}$ & $\mathrm{CNL}$ & 932 & 86.3 & 67.1 \\
\hline & Hv_Contig_947_nlr_1 & $1 \mathrm{~V}$ & $\mathrm{CNL}$ & 948 & 85.7 & 78.1 \\
\hline & Hv_Contig_678_nlr_2 & $1 \mathrm{~V}$ & $\mathrm{CNL}$ & 967 & 85.7 & 68.1 \\
\hline & Hv_Contig_178_nlr_1 & $1 \mathrm{~V}$ & $\mathrm{CNL}$ & 967 & 85.8 & 87.7 \\
\hline & Hv_Contig_1150_nlr_1 & $1 \mathrm{~V}$ & CNL & 937 & 81.3 & 82.4 \\
\hline & Hv_Contig_785_nlr_1 & $1 \mathrm{~V}$ & $\mathrm{CNL}$ & 859 & 85.8 & 69.0 \\
\hline & Hv_Contig_399_nlr_1 & $1 \mathrm{~V}$ & $\mathrm{CNL}$ & 959 & 87.3 & 75.8 \\
\hline & Hv_Contig_422_nlr_1 & $1 \mathrm{~V}$ & $\mathrm{CNL}$ & 943 & 87.5 & 78.0 \\
\hline & Hv_Contig_727_nlr_1 & $1 \mathrm{~V}$ & $\mathrm{CNL}$ & 767 & 84.4 & 70.0 \\
\hline & Hv_Contig_559_nlr_1 & $1 \mathrm{~V}$ & $\mathrm{CNL}$ & 745 & 80.1 & 73.5 \\
\hline & Hv_Contig_238_nlr_1 & $1 \mathrm{~V}$ & $\mathrm{CNL}$ & 964 & 84.8 & 63.9 \\
\hline & Hv_Contig_668_nlr_1 & $1 \mathrm{~V}$ & $\mathrm{CNL}$ & 909 & 84.5 & 85.0 \\
\hline & Hv_Contig_119_nlr_2 & $1 \mathrm{~V}$ & $\mathrm{CNL}$ & 947 & 80.5 & 91.1 \\
\hline & Hv_Contig_924_nlr_1 & $1 \mathrm{~V}$ & $\mathrm{CNL}$ & 947 & 84.9 & 84.9 \\
\hline \multirow[t]{4}{*}{ Pm3b } & Hv_Contig_1461_nlr_1 & $1 \mathrm{~V}$ & $\mathrm{CNL}$ & 1422 & 91.5 & 93.4 \\
\hline & Hv_Contig_1202_nlr_1 & $1 \mathrm{~V}$ & $\mathrm{CNL}$ & 1525 & 90.1 & 100 \\
\hline & Hv_Contig_1215_nlr_1 & $1 \mathrm{~V}$ & $\mathrm{CNL}$ & 1473 & 90.4 & 93.7 \\
\hline & Hv_Contig_1186_nlr_1 & $1 \mathrm{~V}$ & $\mathrm{CNL}$ & 1485 & 84.7 & 96.9 \\
\hline \multirow[t]{3}{*}{ Sr45 } & Hv_Contig_1433_nlr_1 & $1 \mathrm{~V}$ & $\mathrm{CNL}$ & 1204 & 91.6 & 65.2 \\
\hline & Hv_Contig_1007_nlr_1 & $1 \mathrm{~V}$ & CNNL & 1156 & 91.6 & 61.6 \\
\hline & Hv_Contig_232_nlr_1 & $1 \mathrm{~V}$ & $\mathrm{CNL}$ & 1219 & 84.1 & 73.1 \\
\hline \multirow[t]{2}{*}{ Yr7 } & Hv_Contig_909_nlr_1 & $2 \mathrm{~V}$ & CC-zf-BED-NL & 1509 & 80.0 & 82.0 \\
\hline & Hv_Contig_430_nlr_1 & $2 \mathrm{~V}$ & zf-BED-NL & 1417 & 74.5 & 91.3 \\
\hline$R C R 1$ & Hv_Contig_1205_nlr_1 & $3 \mathrm{~V}$ & CNL & 943 & 87.8 & 96.6 \\
\hline \multirow[t]{2}{*}{ Sr35 } & Hv_Contig_191_nlr_1 & $3 \mathrm{~V}$ & $\mathrm{CNL}$ & 922 & 89.5 & 67.0 \\
\hline & Hv_Contig_479_nlr_1 & $3 \mathrm{~V}$ & $\mathrm{CNL}$ & 918 & 84.3 & 78.2 \\
\hline \multirow[t]{3}{*}{ Lr1 } & Hv_Contig_1032_nlr_1 & $5 \mathrm{~V}$ & $\mathrm{CNL}$ & 872 & 86.3 & 73.6 \\
\hline & Hv_Contig_1499_nlr_1 & $5 \mathrm{~V}$ & $\mathrm{NL}$ & 1169 & 86.1 & 71.2 \\
\hline & Hv_Contig_582_nlr_1 & $5 \mathrm{~V}$ & CNL & 1392 & 85.3 & 77.6 \\
\hline
\end{tabular}


Table 3 (continued)

\begin{tabular}{|c|c|c|c|c|c|c|}
\hline & Contig number & $\begin{array}{l}\text { In silico } \\
\text { location }\end{array}$ & Type of NLR & $\begin{array}{l}\text { Length of protein } \\
\text { (aa) }\end{array}$ & $\begin{array}{l}\text { Identity to } \\
\text { reference (\%) }\end{array}$ & $\begin{array}{l}\text { Coverage } \\
\text { (\%) }\end{array}$ \\
\hline \multirow[t]{3}{*}{ Pm2 } & Hv_Contig_335_nlr_1 & $5 \mathrm{~V}$ & $\mathrm{CNL}$ & 1260 & 93.0 & 99.8 \\
\hline & Hv_Contig_1045_nlr_1 & $5 \mathrm{~V}$ & $\mathrm{CNL}$ & 1230 & 86.1 & 89.4 \\
\hline & Hv_Contig_810_nlr_1 & $5 \mathrm{~V}$ & $\mathrm{CNL}$ & 1053 & 80.1 & 86.0 \\
\hline
\end{tabular}

chromosomes of $H$. villosa, however, these $R$ genes also lack orthologues in barley. Our results indicate that orthologues of cloned wheat $R$ genes could be efficiently captured by NLR enrichment, and the data could be used for evolutionary an functional studies of NLR genes at specific loci.

\section{Identification of NLRs with integrated domains}

Atypical domains in NLRs have recently been found to play vital roles in the recognition of pathogens, and these domains can be found in any region of the protein. The additional atypical domains fused into NLRs were designated as integrated decoys (ID), and those proteins with IDs were then classified to be NLR-IDs [12]. Among the 772 determined NLRs of $H$. villosa, a total of 52 NLR-IDs were identified carrying domains other than NB-ARC, CC, and LRR. The identified 15 types of IDs included DDE_Tnp_4, Kelch repeats, Thioredoxin, Pkinase, zfBED, B3 DNA binding, PP2C, WRKY and others (Fig. 4). It was interesting to find that the DDE, Kelch and PP2C domains preferred to fuse with the LRR-terminal, the Zinc Finger-BED preferred to fuse with the CC-terminal, while the thioredoxin and Pkinase domains could fuse with both terminals (Fig. 4).

As shown by phylogenetic analysis based on all the NLRs in Fig. 2, NLRs integrated with DDE_Tnp_4, the largest NLR-ID group, were clustered into one branch as Clade B. However, NLR-IDs containing different domains were clustered into 'Clade J' and 'Clade G' indicating that NLRs in these groups appear to have a predisposition to fuse with other proteins. In addition, the region involved in integration was different in that the NLRs in 'Clade J' tend to fuse decoys in the LRR-terminal, while the NLRs in Clade G tend to fuse decoys in the CC-terminal.

The phylogenetic analysis using 52 NLR-IDs of $H$. villosa was also conducted as shown in Fig. 5 (https:// itol.embl.de/shared/2018201031). Most of the NLRIDs with the same IDs could be phylogenetically clustered, especially those NLR-IDs containing DDE, B3 and Kelch clustered into large groups, respectively. The tightly clustered NLR-IDs with similar domain compositions in the tree were usually in silico located on the same chromosomes, indicating that these members likely expanded by tandem duplication after the NLR fused with the ID. There are exceptions, for example, NLR-IDs with Pkinase were clustered into different groups indicating independent cases of fusion. The direct evidence is that the tightly grouped NLR-IDs, Hv-contig-705, Hv-contig-869 and Hv-contig-1233, contained a Pkinase integrated into the CC-terminal, while Hv-contig-316 and Hv-contig-317 contained a Pkinase integrated into the LRR-terminal (Figs. 4 and 5). Another example for independent fusion concerns the case of RVT_1 which is found integrated into Hvcontig-967, Hv-contig-127 and Hv-contig-663, which have different domain compositions and chromosome locations (Figs. 4 and 5).

The NLR-IDs were extracted from another six Triticeae species, then the number of NLR-IDs and the types of IDs were compared in detail. A total of 65 IDs were detected from the seven species (Table 4). The number of shared IDs and specific IDs in each species were as follows, 28 and 5 in T. aestivum, 35 and 19 in T. urartu, 18 and 2 in Ae. tauschii, 19 and 6 in H. vulgare, 9 and 0 in B. distachyon, and 8 and 3 in O. sativa (Table 4). Among the 15 IDs found in H. villosa, 12 IDs were shared with at least one species. However, three IDs, including DUF948, NAM-associated and PRT_C, specifially existed in $H$. villosa implying the emergence of these after divergence of $H$. villosa. The phylogenetic tree was also constructed using all the NLR-IDs from these species. It was found that NLR-IDs fused with Kelch and B3 domains expanded dramatically in $H$. villosa, however, NLR-IDs containing Pkinase were fewer in number, whereas those containing Jacalin were altogether lacking in $H$. villosa (Fig. 6, https://itol.embl.de/shared/2018201031).

We found that the homeologues of paired NLRs, such as RPS4/RRS1, Rpg5/RGA1, and RGA4/RGA5, usually displayed a pattern of head-to-head arrangement. $N L R$-IDs with physically tightly linked NLRs often form paired protein complexes. In this study, we found that nine NLR-IDs were located tandemly with another NLRs in the same contig, and in two contigs, namely Hv_Contig_48 and Hv_Contig_1157, NLR-IDs and its linked NLRs were arranged in a head-to-head pattern. 


\begin{tabular}{|c|c|c|}
\hline & $\begin{array}{l}\text { Hv_Contig_1272_nlr_1 } \\
\text { Hv_Contig_165_nlr_1 } \\
\text { Hv_Contig_151_nlr_1 } \\
\text { Hv_Contig_48_nlr_2 } \\
\text { Hv_Contig_523_nlr_2 } \\
\text { Hv_Contig_1146_nlr_1 } \\
\text { Hv_Contig_1157_nlr_2 } \\
\text { Hv_Contig_572_nlr_1 } \\
\text { Hv_Contig_433_nlr_1 } \\
\text { Hv_Contig_717_nlr_1 } \\
\text { Hv_Contig_1233_nlr_1 } \\
\text { Hv_Contig_705_nlr_1 } \\
\text { Hv_Contig_869_nlr_2 } \\
\text { Hv_Contig_316_nlr_1 } \\
\text { Hv_Contig_317_nlr_1 } \\
\text { Hv_Contig_716_nlr_1 } \\
\text { Hv_Contig_739_nlr_1 } \\
\text { Hv_Contig_489_nlr_1 } \\
\text { Hv_Contig_124_nlr_1 } \\
\text { Hv_Contig_813_nlr_1 } \\
\text { Hv_Contig_663_nlr_1 } \\
\text { Hv_Contig_127_nlr_1 } \\
\text { Hv_CContig_967_nlr_1 } \\
\text { Hv_Contig_1485_nlr_1 } \\
\text { Hv_Contig_1469_nlr_1 } \\
\text { Hv_Contig_438_nlr_1 } \\
\text { Hv_CContig_61_nlr_1 }\end{array}$ & 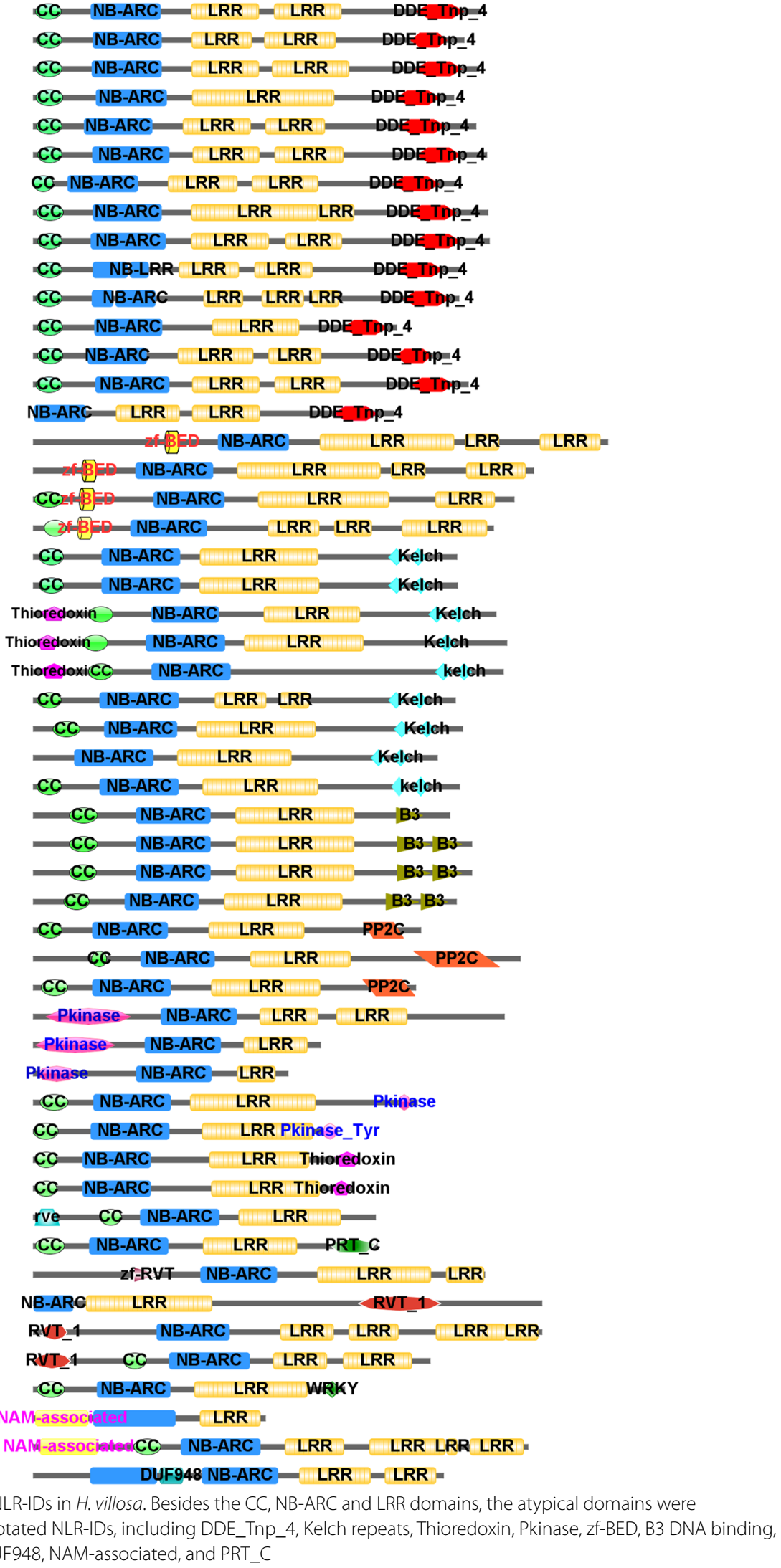 \\
\hline
\end{tabular}




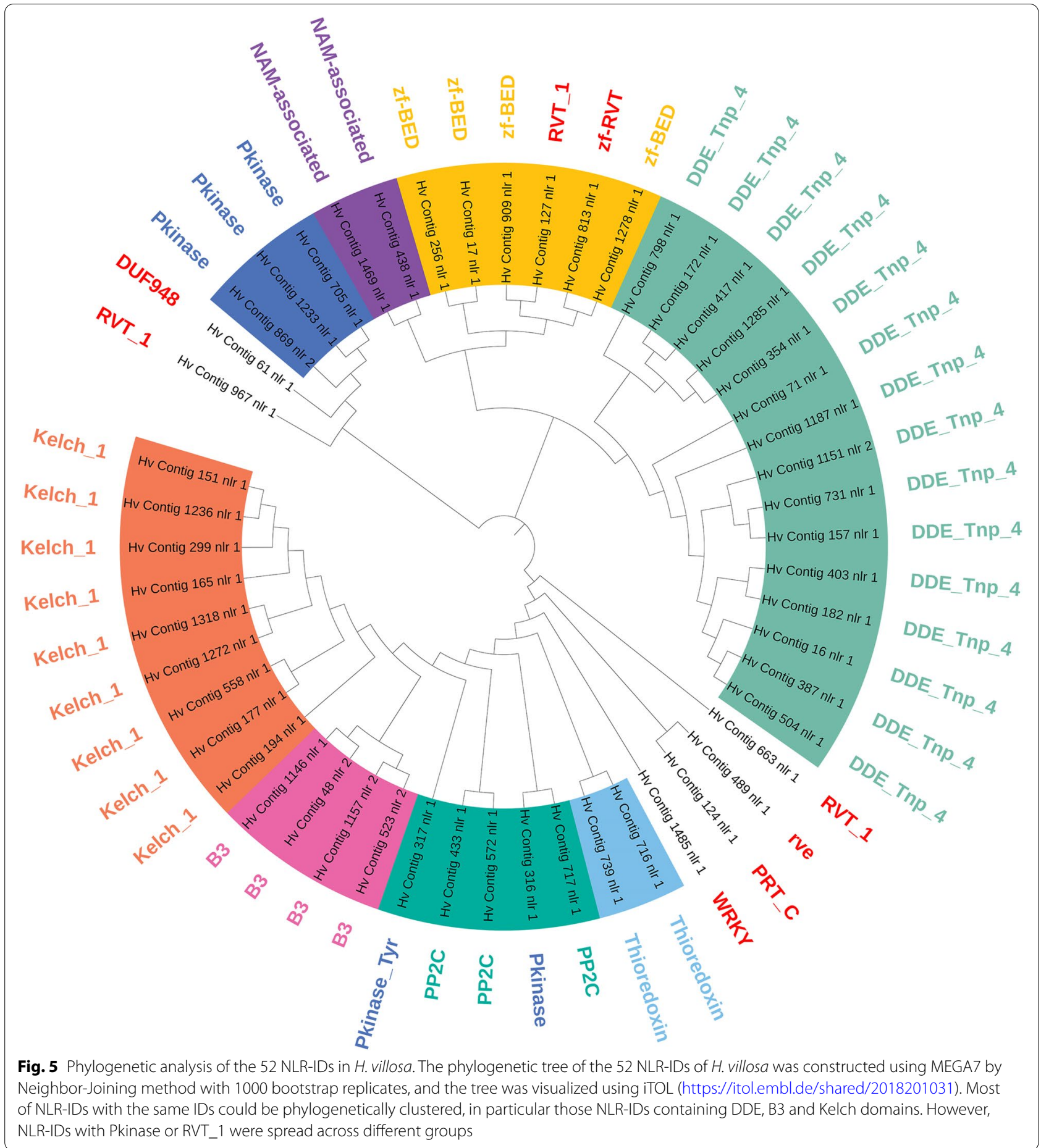

\section{Discussion}

\section{SMRT-RenSeq and NLR-annotator facilitates reference-free} genome-wide mining of NLRs in a wild grass species

NLRs display high domain-conservation even across species. Previously, random isolation of NLRs from whole genomes was achieved using homology-based PCR in wide variety of species [8]. In recent years, great progress has been made in whole genome NLR gene discovery in species with reference quality genomes, but less so in the large number of non-sequenced species. RenSeq, however, has greatly promoted reference-free genome-wide identification of NLRs and accelerated the cloning of 
Table 4 The integrated domains identified in seven grass species

\begin{tabular}{|c|c|c|}
\hline Integrated Domain & Description of IDs & Genomes where IDs were detected \\
\hline Thioredoxin & Thioredoxin & VV, AABBDD, AA, DD, HH, Bd, Os \\
\hline B3 & B3 DNA binding domain & W, AABBDD, AA, DD, HH, Os \\
\hline Pkinase & Protein kinase domain & W, AABBDD, AA, DD, HH, Bd \\
\hline Pkinase_Tyr & Protein tyrosine and serine/threonine kinase & W, AABBDD, AA, DD, HH, Bd \\
\hline WRKY & WRKY DNA-binding domain & W, AABBDD, AA, DD, HH, Bd \\
\hline zf-BED & BED zinc finger & W, AABBDD, AA, DD, HH, Bd \\
\hline DDE_Tnp_4 & DDE superfamily endonuclease & W, AABBDD, DD, HH, Bd \\
\hline PP2C & Protein phosphatase $2 \mathrm{C}$ & W, AABBDD, AA, DD, HH \\
\hline Kelch_1 & Kelch motif & W, AABBDD, AA, DD, HH \\
\hline RVT_1 & Reverse transcriptase & W, AA \\
\hline rve & Integrase core domainV & $\mathrm{W}, \mathrm{AA}$ \\
\hline zf-RVT & Zinc-binding in reverse transcriptase & $\mathrm{W}, \mathrm{AA}$ \\
\hline DUF948 & Domain of unknown function & W \\
\hline NAM-associated & EF-hand domain pair & W \\
\hline PRT_C & NPR1/NIM1 like defence protein $C$ terminal & W \\
\hline Jacalin & Jacalin-like lectin domain & $\mathrm{AABBDD}, \mathrm{DD}, \mathrm{HH}, \mathrm{Bd}, \mathrm{Os}$ \\
\hline DUF761 & Cotton fibre expressed protein & $\mathrm{AABBDD}, \mathrm{DD}, \mathrm{Os}$ \\
\hline Exo70 & Exo70 exocyst complex subunit & $\mathrm{AABBDD}, \mathrm{AA}, \mathrm{HH}$ \\
\hline Motile_Sperm & MSP (major sperm protein) domain & $A A B B D D, A A, D D$ \\
\hline CG-1 & CG-1 domains & $A A B B D D, A A$ \\
\hline DUF295 & Unknown function & $A A B B D D, A A$ \\
\hline Ank_2 & Ankyrin repeats & $\mathrm{AABBDD}, \mathrm{AA}$ \\
\hline TB2_DP1_HVA22 & TB2/DP1, HVA22 family & AABBDD, DD \\
\hline DUF296 & Plants and prokaryotes conserved (PCC) domain & AABBDD, DD \\
\hline GRAS & GRAS (GAI, RGA, SCR) family & AABBDD, DD \\
\hline Myb_DNA-binding & Myb-like DNA-binding domain & AABBDD, Bd \\
\hline AP2 & AP2 domain & $\mathrm{AABBDD}, \mathrm{Bd}$ \\
\hline RIP & Ribosome inactivating protein & AABBDD, $\mathrm{HH}$ \\
\hline VQ & VQ motif & AABBDD, Os \\
\hline AvrRpt-cleavage & Cleavage site for pathogenic type III effector avirulence factor Avr & $\mathrm{DD}, \mathrm{HH}$ \\
\hline BPS1 & Staphylococcal nuclease homologue & AABBDD \\
\hline CPSF100_C & Tudor domain & AABBDD \\
\hline Ceramidase & Bacterial protein of unknown function & AABBDD \\
\hline TIG & No apical meristem-associated C-terminal domain & AABBDD \\
\hline zf-RING_2 & Phosphoribosyltransferase C-terminal & AABBDD \\
\hline Aldo_ket_red & AUX/IAA family & AA \\
\hline BTB & FNIP Repeat & AA \\
\hline CwfJ_C_1 & Glutaredoxin & AA \\
\hline CwfJ_C_2 & Paired amphipathic helix repeat & AA \\
\hline DUF3420 & Zinc-finger of the FCS-type, C2-C2 & AA \\
\hline DUF3615 & LSD1 zinc finger & AA \\
\hline DUF4216 & C1 domain & AA \\
\hline DUF4218 & F-box & AA \\
\hline EF-hand_7 & Transport inhibitor response 1 protein domain & AA \\
\hline NPR1_like_C & Protein BYPASS1-related & AA \\
\hline PARP & Cleavage and polyadenylation factor 2 C-terminal & AA \\
\hline PTEN_C2 & Ceramidase & AA \\
\hline RST & IPT/TIG domain & AA \\
\hline RVT_3 & Ring finger domain & AA \\
\hline
\end{tabular}


Table 4 (continued)

\begin{tabular}{lll}
\hline Integrated Domain & Description of IDs & Genomes where IDs were detected \\
\hline Retrotran_gag_2 & Aldo/keto reductase family & AA \\
XH & BTB/POZ domain & AA \\
gag_pre-integrs & Protein similar to CwfJ C-terminus 1 & AA \\
tRNA_synt_2f & Protein similar to CwfJ C-terminus 2 & AA \\
zf-CCHC_4 & Domain of unknown function & AA \\
SNase & Protein of unknown function & DD \\
TUDOR & Domain of unknown function & DD \\
AUX_IAA & Poly (ADP-ribose) polymerase catalytic domain & HH \\
FNIP & C2 domain of PTEN tumour-suppressor protein & $H H$ \\
Glutaredoxin & RCD1-SRO-TAF4 (RST) plant domain & $H H$ \\
PAH & Reverse transcriptase-like & $H H$ \\
zf-FLZ & Gag-polypeptide of LTR copia-type & $H H$ \\
zf-LSD1 & XH domain & $H H$ \\
C1_2 & GAG-pre-integrase domain & Os \\
F-box_5 & Glycyl-tRNA synthetase beta subunit & Os \\
Transp_inhibit & Zinc knuckle & Os \\
\hline
\end{tabular}

Note: $V$ indicates species Haynaldia villosa, AABBDD indicates Triticum aestivum, AA indicates Triticum urartu, DD indicates Aegilops tauschii, HH indicates Hordeum vulgare, $B d$ indicates Brachypodium distachyon and Os indicates Oryza sativa

disease resistance genes efficiently from non-model and non-crop species. The application of RenSeq in Solanum tuberosum, a wild potato, indicated that $\sim 80 \%$ sequence identity is enough for capturing NLR genes using oligonucleotide baits [34]. Previously, we compared $H$. villosa and barley and found a high degree of identity between these two closely related species [11]. We therefore used a barley NLR bait library in this study to capture NLRs from $H$. villosa. The enrichment efficiency analysis using the sequences of cloned $R$ genes indicated that the homologous genes in $H$. villosa were successfully enriched. Therefore, the bait library designed based on a species with ample genomic resources could be used to isolate NLRs from an evolutionary closely related species.

Before annotation of the NLRs from the assembled contigs, the redundant contigs were removed using the cutoff value of 90 and $95 \%$. Actually, the same 53 contigs were removed by both parameters. To avoid the situation that a recent duplication or residual heterozygosity perhaps could be ruled out as a source for some of the removed contigs, these 53 removed contigs were reanalyzed (Table S4). It was found that the 22 contigs contained no, partial or pseudogene NLRs, 16 contigs contained complete NLRs with $>99 \%$ identity to the already annotated NLRs, and the 15 contigs contained complete NLRs with $95-99 \%$ identity to the already annotated NLRs. Finally, the contigs with no, partial, pseudogene NLRs or with complete NLRs showing >99\% identity to the already annotated NLRs were removed. The reannotated 15 complete NLRs showing 95-99\% identity to the already annotated NLRs were included in the database along with the previously identified 772 NLRs.

\section{RenSeq helps to identify more NLRs in Haynaldia villosa}

NLR complements have been identified in several diploid species related to $H$. villosa using different reference assemblies and different pipelines, such as 463, 570, 563 or 558 NLRs in T. urartu, 842 or 738 NLRs in A. tauschii, 420, 336 or 462 NLRs in H. vulgare, 422 or 341 NLRs in $S$. bicolor, and 470 or 438 in S. italica [4, 22, 44, 63]. In this study, using the latest version of the released genomic database, we identified 350 NLRs from B. distachyon, 347 from O. sativa, 397 from $H$. vulgare, 530 from T. urartu, 572 from $A$. tauschii and 772 from $H$. villosa by the same pipeline. $H$. villosa contained relatively more NLRs than what has been reported in most diploid genomes. The study in Solanum tuberosum indicated that more NLRs were revealed by RenSeq than those predicted by the then available annotation software and genome reference suggesting that RenSeq facilitates the recovery of NLRs from the poorly or previously unannotated regions of the genome [34]. Moreover, cDNA SMRT-RenSeq, with its longer reads, could help correct splicing errors generated by using short read sequencing technology. The large number of NLRs that we recorded in our study in $\mathrm{H}$. villosa, might be accounted for by the NLR expansion that occurred at some loci.

Some of the in silico located NLRs were selected for physical location to specific chromosome arms, and good 

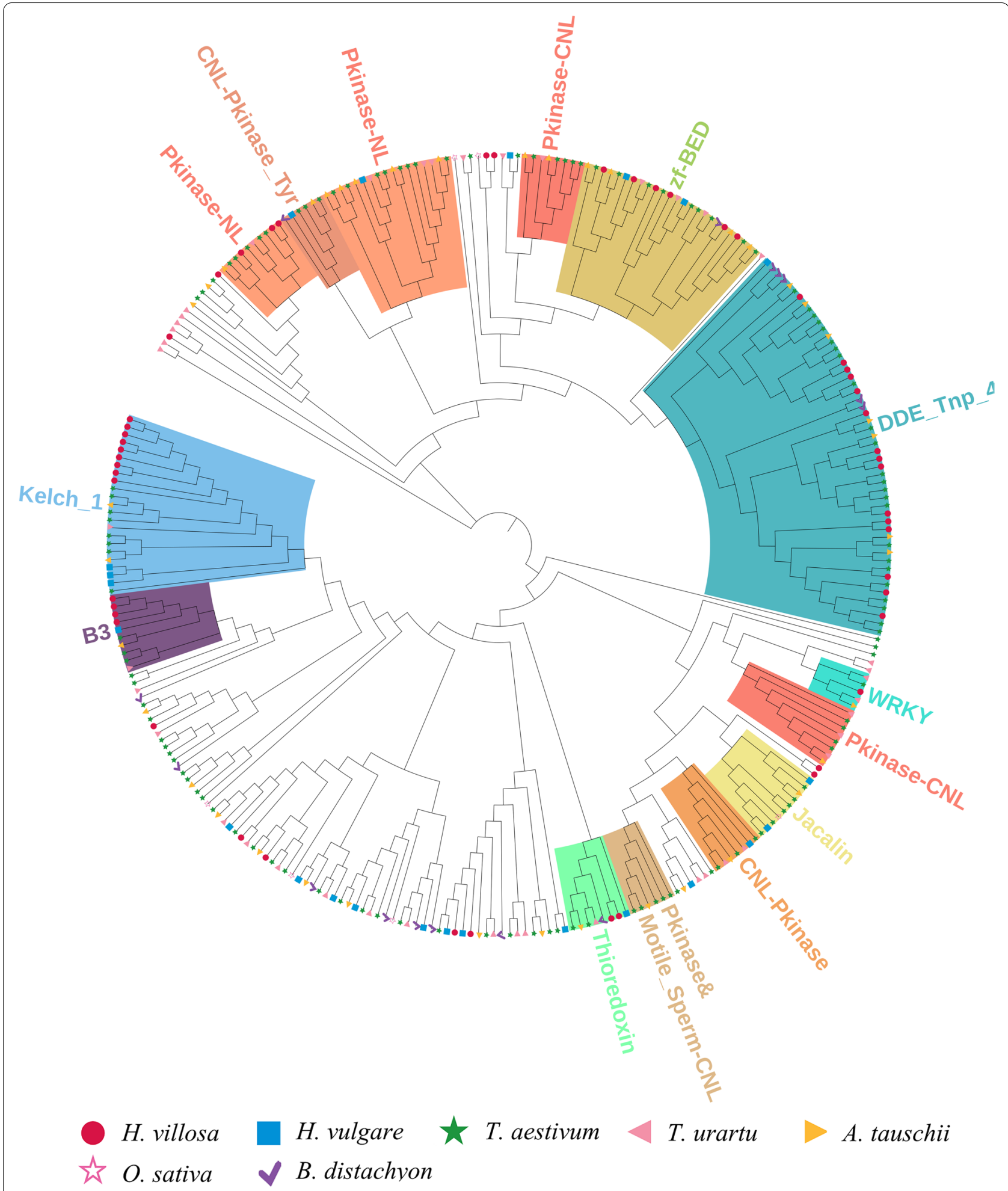

Fig. 6 Phylogenetic analysis of the 315 NLR-IDs in seven Triticeae species. The phylogenetic tree of the 315 NLR-IDs from seven Triticeae species was constructed using MEGA7 by Neighbor-Joining method with 1000 bootstrap replicates, and the tree was visualized using iTOL (https://itol.embl.de/ shared/2018201031). NLR-IDs carrying Kelch and B3 domains showed a pronounced expansion in H. villosa, whereas NLR-IDs with Pkinase were less abundant, and those with Jacalin were altogether lacking 
congruency was found for chromosomes $1 \mathrm{~V}, 2 \mathrm{~V}, 3 \mathrm{~V}, 5 \mathrm{~V}$ and $6 \mathrm{~V}$ suggesting these NLRs could be used as candidates for mining for $\mathrm{R}$ genes genetically assigned to these chromosomes. However, some of the NLRs, which were in silico located on $7 \mathrm{VS}$ were physically located on $4 \mathrm{VL}$. Therefore, NLRs in silico located on 7 VS could be used as candidates for mining for $\mathrm{R}$ genes which map to $4 \mathrm{VL}$. Chromosomal rearrangements are commonly found in wheat and its wild relatives [75], and our results will facilitate accurate $\mathrm{R}$ gene location and cloning in such chromosome regions.

\section{NLRs with unique integrated domains are found in Haynaldia villosa}

In host-pathogen interactions, a decoy is used to describe those molecules which mimic a component manipulated during infection [72]. The decoy strategy is used by both the pathogen to inhibit the host defense and by the host to inhibit the pathogen infection [55]. In the host, decoys are often integrated with NLRs, but decoys can also be physically independent of the NLRs [17]. For example, Pto in tomato mimics the RLKs that are targeted by the effector AvrPto injected into the host cell by the bacterial pathogen Pseudomonas syringae. The partner of Pto, Prf, an NLR receptor, senses the modification of Pto by AvrPto to trigger immune signaling [51]. Sometimes, decoys, referred to as integrated-decoys, are fused with the $\mathrm{N}$ or $\mathrm{C}$ terminal of NLRs. For example, RRS1, an integrated-decoy NLR from $A$. thaliana, carries a C-terminal WRKY domain which mimics the WRKY protein targeted by the PopP2 effector. Other examples include the NLRs RGA5 and Pik-1 in rice, which carry a heavy metal associated (HMA) domain targeted by AVR-Pia and AVR-Pik, respectively [13, 53, 82]. Intracellular detection of pathogen-derived molecules through integrated domains in NLRs is a typical resistance pathway among the nine mechanisms identified in hosts to date [36].

Integration of decoy domains in NLRs is frequent in plants. It was found that on average across 31 species analyzed, $3.5 \%$ of all NLR proteins are integrated with 90 diverse protein domains, with $2.5 \%$ in CNLs and $4.7 \%$ in TNLs [37]. However, Sarris [63] predicted that as many as $10 \%$ of plant NLRs contain highly variable domains. In the present study, 52 NLR-IDs (about 6.7\%) were found among the 772 NLRs in $H$. villosa. While the NLR-ID ratio was smaller in $H$. villosa compared to $T$. urartu and Ae. tauschii, the total number of NLR-IDs in H. villosa was the largest among all the seven species studied (Table 4). Except for B. distachyon, unique IDs were detected in each species, indicating that novel fusions happened after species divergence. The most cases were observed in T. urartu harboring 19 distinct IDs, while three cases happened in $H$. villosa harboring three distinct IDs. We also found 'Clade J' and 'Clade G' of $H$. villosa appear to have a predisposition to fuse with other proteins, and a major integration clade whose members underwent repeated independent integration events was also described [4].

The decoys integrated in NLRs are usually the duplicated products of effector targets. Protein kinases, transcription factors and proteases, which have been reported to be effector targets previously, were found to be fused as decoys in NLRs with high frequency. Therefore, genome-wide mining of NLR-IDs can help identify putative effector targets. The integrated domains identified here in $H$. villosa include DDE_Tnp_4, kelch repeats, thioredoxin, protein kinase, zinc finger-BED, B3 DNA binding, integrase and $\mathrm{PP} 2 \mathrm{C}$. These protein families are therefore considered as likely effector targets.

Previous studies have found that NLR-IDs usually work in pairs and link proximally in genome. For example, RPS4 works in concert with RRS1 to confer resistance to P. syringae [53], RPP2A pairs with RPP2B to confer resistance to Hyaloperonospora arabidopsidis [65], Rpg5 pairs with RGA1 to confer resistance to $P$. tritici [74], while Pikh-1 with Pikh-2 [82] and Pi5-1 with Pi5-2 [39] to confer resistance to $M$. oryzae. Therefore, the ability to detect tightly-linked NLRs in genomes can provide important clues as to their modus operandi. In this study, we found nine NLRs-ID located physically proximal to another NLR in the same contig, among which two cases were arranged in a head-to-head orientation. However, NLR-IDs seem not to be physically paired NLRs at the DNA sequence level which was also predicted from a study in rice [37].

\section{Genome-wide exploration and chromosomal location of NLRs is critical for cloning and introducing new resistance genes from $H$. villosa}

Wild crop relatives represent an important reservoir of disease resistance genes, which in some cases can be introduced into cultivated crops by wide crosses. Genetically diverse accessions of $H$. villosa has been configured and shown to display variation in disease resistance [14, $16,26,43,52,59,83-85]$. However, as is the case with most wild species, gene utilization is inefficient. In this study, the genome-wide identification of NLRs from $H$. villos $a$ and mapping of NLRs to each chromosome arm of $H$. villosa will change the way of introducing new resistances into wheat. Firstly, the public availability of all the NLRs provides a precious resource for accelerating the cloning of resistance genes, which will help to introduce new resistances into wheat through gene transformation. Secondly, the information of NLRs sequences and their chromosome locations provides important data for different research groups to compare the NLRs sequences 
located on specific chromosome or even on specific chromosome region between different $H$. villosa accessions, which could help researchers identify new resistance germplasm and transferring new resistance genes or alleles of H.villosa into wheat. Furthermore, the availability of all the NLRs facilitates developing molecular markers specific to H.villosa based on the NLRs, so it could help transfer chromatin containing putative resistance genes of H.villosa into wheat by molecular marker assistant selection and help accelerate developing new resistance germplasms with small segments introgression.

Previously, the SMRT-RenSeq data of $H$. villosa accession 91C43 helped us to clone the broad-spectrum powdery mildew resistance gene $P m 21$ [79]. Following this, alleles of $P m 21$ were cloned by homology-based PCR from more than 100 accessions. The high genetic diversity between these alleles provides valuable information to understand the structure, function and evolution of $P m 21$.

NLRs are subject to intensive diversifying selection due to their genetic interaction with rapidly evolving pests and pathogens $[23,64]$. This process may lead to the production of large amounts of pseudogenes. In $H$. villosa, besides the 772 complete NLRs, we detected another 289 NLRs which were classified as pseudogenes. These were widely spread across the genome and may be the remnants of past evolutionary activity. In the hexaploid wheat cultivar Chinese Spring, there are 1540 complete NLRs and 2360 putative pseudogene NLRs [69]. Pseudogenes may retain useful evolutionary potential through the propensity of NLRs for intergenic recombination. For example, a functional chimeric allele of the leaf rust resistance gene $L r 21$ was recovered from a cross between two non-functional alleles [24]. Moreover, a locus containing a pseudogene in one accession may harbor a functional allele in another accession [47]. For example, the $P m 2$ allele in Chinese spring contains a premature stop codon, while in cultivar Ulka the open reading frame is retained giving rise to a functional resistance gene $[62,69]$. Therefore, the pseudogenes identified in H. villosa may provide valuable clues for mining of functional alleles in other accessions.

\section{Conclusions}

In this study, the genome-wide $N L R$ complement of $H$. villosa was efficiently identified using SMRT-RenSeq, and a total of 772 complete NLRs were annotated. The information of the chromosome location of all the NLRs were provided, which is valuable for resistance gene mining from the specific chromosome. The physical location of NLRs from group 1, 2, 3, 5 and 6 showed a perfect homoeologous relationship with other Triticeae species except the NLRs on chromosome $4 \mathrm{VL}$ which were predicted to be located on the homoeologous group 7 in silico. Cluster expansion observed in some specific gene loci indicated that independent evolutionary cases occurred in $H$. villosa. We also identified 52 NLR-IDs with fifteen types of integrated domains (IDs), among which Kelch and B3-type NLR-IDs experienced expansion, and three type of IDs were unique in $H$. villosa. This study gave an example to successfully capture the genome-wide NLRs in wild species using the baits from another species in Triticeae. The availability of the NLRs from $H$. villosa provides a valuable library for mining and transferring of disease resistance into wheat.

\section{Materials and methods}

\section{NLR-enrichment library construction and physical mapping of NLR genes}

Haynaldia villosa (genome constitution VV, $2 \mathrm{n}=14$ ), with long hairs on the keel of the glume and apex of the lemma, is an annual diploid species which belonging to Triticinae of Triticeae in Gramineae. It is a vigorous ruderal wild plant growing on the harsh, moisture-stressed soils in the northeastern part of the Mediterranean region and Caucasus area [20]. This species usually confers resistance to different diseases and tolerance to various abiotic stresses [20]. The $H$. villosa accession $91 \mathrm{C} 43$ was obtained from Cambridge University, UK and propagated by artificial bagging and self-pollination for $6-7$ successive generations to obtain an inbred line. Tissue from the 91C43 inbred line was used for DNA extraction. This accession was also used as the donor to develop 14 wheat-H.villosa translocation lines through wide-crosses and chromosome engineering, each involving one of the 14 chromosome arms of $H$. villosa, respectively [86]. This set of translocation lines was used for chromosomal location of NLRs.

\section{Library construction for NLR gene enrichment and SMRT sequencing}

The barley NLR bait library TSLMMHV1 [9] was used to capture the NLR complement from $H$. villosa accession 91C43. The design of this NLR bait library has been previously described [9]. In brief, NLR-Parser [ 67], a tool for high-throughput identification of NLRs based on conserved motifs [33], was used to search eight barley tanscriptomes and six barley genomes [25] (IBGSC2012) to identify those sequences containing at least one $\mathrm{CC}$ and two NBS motifs, or two NBS and one LRR motifs [9]. Previously-reported barley disease resistance genes, including Mla, Mlo, Rpg1, etc., were manually added. Repetitive and redundent sequences were removed to yield the final set of 99,421,100-mer RNA baits.

Genomic DNA for SMRT library preparation was extracted from seedlings of $H$. villosa with the DNeasy 
Plant Mini Kit (Qiagen, Hilden, Germany). SMRT library preparation and NLR capture followed the procedure described by Witek et al. [76]. The enriched library was sequenced on the PacBio RSII platform using four SMRT cells in the Genome Analysis Center (TGAC, Norwich Research Park, UK) with P4-C6 chemistry to minimize errors.

\section{Assembly of the SMRT-RenSeq reads}

The raw reads were screened by the SMRT Portal software with parameter settings $>3$ full passes and $>90 \%$ accuracy to generate the inserted sequence ROI. The long ROI were analyzed and assembled using genetic R8 software (www.geneious.com) with defaults of $1 \%$ mismatch, $1 \%$ gap (no more than $3 \mathrm{bp}$ ) and minimum read length overlap greater than $100 \mathrm{nt}$ with at least $98 \%$ identity. Only contigs assembled from at least five sequences and having a minimum coverage of two were considered for further analysis. A total of 1509 contigs fulfilling these criteria were obtained.

\section{Sequence annotation of the assembled SMRT-RenSeq contigs by NLR-annotator}

To obtain non-redundant NLR-containing contigs, the raw assembled contigs were analysed by the global alignment program Cd-hit (version 4.8.1) [42] to construct a phylogenetic tree and remove the sequences with $>95 \%$ identity. The retained contigs were analysed with RepeatMasker followed by screening for NLRs using NLR-Annotator (https://github.com/steuernb/NLR-Annotator).

\section{Extraction of protein sequences from the annotated NLRs}

The predicted NLR loci were extended backwards by $3000 \mathrm{bp}$ from the left border and forwards by $3000 \mathrm{bp}$ from the right border, respectively. The extended sequences were cut out for BLASTx against the database of barley (ftp://ftp.ensemblgenomes.org/pub/plants/relea se-46/fasta/hordeum_vulgare), Aegilops tauchii (ftp://ftp. ensemblgenomes.org/pub/plants/release-46/fasta/aegil ops_tauschii), and common wheat (ftp://ftp.ensemblgen omes.org/pub/plants/release-46/fasta/triticum_aestivum) to find the orthologous genes from the three species. In each species, the proteins with the highest identity and longest coverage were selected as the putative orthologous proteins. The protein sequence from the species with the highest identity and longest coverage compared with $H$. villosa was used as the reference to predict the coding sequence of the corresponding $H$. villosa NLR using FGENESH+ (http://www.softberry.com/).

\section{Domain composition analysis of the annotated NLRs}

The protein sequences of six species, including $T$. aestivum, Ae. tauschii, T. urartu, $H$. vulgare, O. sativa, and $B$. distachyon, were downloaded from Ensembl Plants (http://plants.ensembl.org/info/website/ftp/index.html) and the longest proteins corresponding to the longest transcripts of genes with alternative splicing were used for annotation analysis (Table S1). Plant_rgene (https:// github.com/krasileva/plant_rgenes) and InterProScan (interproscan-version 5.30-69.0) were used to analyze the NLRs annotated as being complete. Plant_rgene program is a pipeline for analyzing the domain composition of putative NLRs, however, sometimes it cannot accurately output LRR domains. The InterProScan software [30] is a complementary tool for identification of the LRR domains deposited in the SUPERFAMILY database. Therefore, all the proteins, including the 774 complete $H$. villosa NLRs and the proteomes from the six species listed above, were analyzed by the Plant_rgene software (evalue $=1 \mathrm{e}-3$ ) to search for conserved domains (including CC, NB-ARC and integrated domains). Then the same set of proteins were analyzed again by InterProScan to search for the conserved LRR domains deposited in the SUPERFAMILY database. The conserved domains output from both programs were integrated. Only those proteins containing an NB-ARC domain were designated as NLRs. If any atypical domain was additionally predicted in the NLRs, these domains were considered to be the integrated-decoys (IDs), and those NLRs containing ID(s) were then designated as NLR-IDs. The diagrams of all the 52 NLR-IDs were displayed by IBS (Version 1.0.3) [45].

\section{Phylogenetic tree construction}

For construction of the phylogenetic tree of all the 772 complete NLRs, the conserved NB-ARC domain was extracted from each NLR and a phylogenetic tree was constructed using MEGA7 by Neighbor-Joining method with 1000 bootstrap replicates [38]. The tree was visualized using iTOL [40]. The conserved motif was searched using MAST (version 4.9.1) [5] based on the 'motif 1' to 'motif 20' defined by Jupe [33], and displayed in the phylogenetic tree. The phylogenetic tree of the 52 detected NLR-IDs in $H$. villosa and all the 315 NLR-IDs from seven Triticeae species were also constructed by the same method using the same parameters.

Identification of the orthologous NLR genes corresponding to cloned $R$ genes

The cloned $R$ genes, including Mla1 (AY009939.1), Sr50 (KT725812.1), Sr35 (KC573058.1), Pm3b (AY325736.1), RCR1 (KU161103.1), Lr1 (EF439840.1) and $Y r 7$ 
(MN273771.1) were downloaded from the NCBI database, then the nucleotide sequences were used to perform a sequence alignment with the assembled SMRT RenSeq contigs. The sequences showing $>80 \%$ identity and $>60 \%$ coverage with the cloned $\mathrm{R}$ genes were screened from the annotated NLRs. Then the screened NLRs in silico located to the same homoeologous chromosome loci as the corresponding queries were considered as their putative orthologues respectively.

\section{The chromosomal location of NLRs}

The nucleotide sequences of the identified NLRs were used as query for BLASTn analysis against the genome databases of barley (ftp://ftp.ensemblgenomes.org/pub/plants/ release-46/fasta/hordeum_vulgare), Aegilops tauchii (ftp:// ftp.ensemblgenomes.org/pub/plants/release-46/fasta/ aegilops_tauschii), Triticum urartu (ftp://ftp.ensemblgen omes.org/pub/plants/release-46/fasta/triticum_urartu) and common wheat (ftp://ftp.ensemblgenomes.org/pub/ plants/release-46/fasta/triticum_aestivum) to locate NLRs to chromosomes. Then, a subset of the NLRs were selected to determine the physical location by PCR analysis using 14 wheat- $H$. villosa translocation lines each involving one of the 14 chromosome arms of $H$. villosa. The PCR primers were designed according to specific deletions or insertions in the $H$. villosa NLRs when compared to the presumed orthologous genes in Chinese Spring (Table S2). The PCR procedure was as follows: incubation at $94^{\circ} \mathrm{C}$ for $3 \mathrm{~min}$ followed by 33 cycles of $94^{\circ} \mathrm{C}$ for $45 \mathrm{~s}, 55^{\circ} \mathrm{C}-60^{\circ} \mathrm{C}$ for $45 \mathrm{~s}$, and $72^{\circ} \mathrm{C}$ for $1 \mathrm{~min}$. The PCR products were separated by polyacrylamide gel electrophoresis (Acrylamide:Bisacry lamide $=39: 1$ ) followed by silver nitrate staining.

\section{PacBio transcriptome sequencing of $H$. villos $a$ and NLR expression analysis}

PacBio sequencing of the full-length transcriptome extracted from ten mixed tissues of $H$. villosa accession $91 \mathrm{C} 43$ was performed by Novogene (Beijing). The mixed tissue sample included seedling leaves, seedling stems, seedling roots, immature spikes, seedling leaves inoculated with Bgt for 6 and 24h, seedling leaves and roots treated with $200 \mathrm{mM} \mathrm{NaCl}$ for 3 days, and seedling leaves and roots treated with $16 \%$ PEG4000 for 3 days. The PacBio trasncriptome sequences were screened by NLRAnnotator. Then the annotated NLRs of SMRT RenSeq were used to search against the annotated transcribed NLRs. When an NLR from SMRT RenSeq could find a corresponding transcribed NLR with an identity higher than $95 \%$ and coverage longer than $90 \%$, then the enriched NLR was considered to be expressed. The sequences of all the expressed NLRs were compared with their genomic counterparts one by one to analyze the accuracy of the protein prediction based on the genomic sequence.

\section{Abbreviations}

NLR: Nucleotide-binding and leucine-rich repeat; SMRT: PacBio singlemolecule real-time; RenSeq: Resistance gene enrichment sequencing; IDs: Integrated domains; NLR-IDs: NLR with integrated domains; CC: Coiled-coil domain; NB-ARC: NB-ARC domain; LRR: Leucine-rich-repeat domain; PAMPS: Pathogen associated molecular patterns; PRRs: Pattern-recognition receptors; PTI: PAMP-triggered immunity; ETI: Effector-triggered immunity.

\section{Supplementary Information}

The online version contains supplementary material available at https://doi. org/10.1186/s12864-022-08334-w.

\section{Additional file 1. \\ Additional file 2. \\ Additional file 3. \\ Additional file 4. \\ Additional file 5 . \\ Additional file 6 . \\ Additional file 7.}

Additional file 8.

\section{Acknowledgements}

This research supported by the high-performance computing platform of Bioinformatics Center, Nanjing Agricultural University. We highly appreciate Dr. Kamil Witek and Prof Jonathan D. G. Jones kind help in the design and performance of SMRT-RenSeq experiment, and Dr. Matthew Moscou (The Sainsbury Laboratory, UK) for sharing the barley baits library.

\section{Authors' contributions}

$L X$ and $A C$ participated in the design of the experimental plan. $Z \mathrm{H}$ and $Z L$ performed bioinformatics analysis. J L, Y L and R Z performed cytogenetic stocks development. P H, BY and F Q performed NLRs specific marker analysis. A C, L X, P C and BW wrote the manuscript. All authors have read and approved the final manuscript.

\section{Funding}

This work was supported by Natural Science Foundation of China (Grant No. $31671685,31771779)$, the Important National Science \& Technology Specific Projects of Transgenic Research (Grant No. 2018ZX0800905B) and Postgraduate Research \& Practice Innovation Program of Jiangsu Province (Grant No. SJKY19_0541). BW was supported by the Biotechnology and Biological Sciences Research Council cross-institute strategic program Designing Future Wheat (BB/P016855/1).

\section{Availability of data and materials}

The H. villosa SMRT-RenSeq data is available from NCBI under study number SRP132107(https://www.ncbi.nlm.nih.gov/sra/?term=SRP132107). The data of coding sequences and the protein sequences of the annotated NLRs are available in NCBI Genebank database with accession number MZ672249MZ673036. The data of the full-length transcriptome of H. villosa with PacBio SMRT chemistry is available in NCBI SRA database with the accession number SRR15206288 (https://www.ncbi.nlm.nih.gov/sra/SRR15206288). The protein data used for NLR identification from different species were provided in the supplementary information file Table S1, which were obtained from Ensembl Plants database, including: Aegilops tauschii (ftp://ftp.ensemblgenomes. org/pub/plants/release-46/fasta/aegilops_tauschii/pep/), Brachypodium distachyon (ftp://ftp.ensemblgenomes.org/pub/plants/release-46/fasta/brach ypodium_distachyon/pep/), Hordeum vulgare (ftp://ftp.ensemblgenomes. org/pub/plants/release-46/fasta/hordeum_vulgare/pep/), Oryza sativa (ftp:// ftp.ensemblgenomes.org/pub/plants/release-46/fasta/oryza_sativa/pep/), Triticum aestivum (ftp://ftp.ensemblgenomes.org/pub/plants/release-46/fasta/ triticum_aestivum/pep/) and Triticum urartu (ftp://ftp.ensemblgenomes.org/ pub/plants/release-46/fasta/triticum_urartu/pep/). In addition, the genomic data used to help identify and chromosomal locate of NLR from H.villosa were avalible in Ensembl Plants database, including Aegilops tauschii (ftp://ftp. 
ensemblgenomes.org/pub/plants/release-46/fasta/aegilops_tauschii/dna/), Hordeum vulgare (ftp://ftp.ensemblgenomes.org/pub/plants/release-46/fasta/ hordeum_vulgare/dna/) and Triticum aestivum (ftp://ftp.ensemblgenomes. org/pub/plants/release-46/fasta/triticum_aestivum/dna/).

\section{Declarations}

Ethics approval and consent to participate

Not applicable.

\section{Consent for publication}

Not applicable.

\section{Competing interests}

The authors declare that they have no competing interests.

\section{Author details}

${ }^{1}$ National Key Laboratory of Crop Genetics and Germplasm Enhancement, Cytogenetics Institute, Nanjing Agricultural University/CIC-MCP, Nanjing 210095, China. ${ }^{2}$ John Innes Centre, Norwich Research Park, Norwich NR4 7UH, UK. ${ }^{3}$ Center for Desert Agriculture, Biological and Environmental Science and Engineering Division (BESE), King Abdullah University of Science and Technology (KAUST), Thuwal 23955-6900, Saudi Arabia.

Received: 12 March 2021 Accepted: 24 January 2022

Published online: 10 February 2022

\section{References}

1. Ames N, Dreiseitl A, Steffenson BJ, Muehlbauer GJ. Mining wild barley for powdery mildew resistance. Plant Pathol. 2015;64(6):1396-406.

2. Arora S, Steuernagel B, Gaurav K, Chandramohan S, Long Y, Matny O, et al. Resistance gene cloning from a wild crop relative by sequence capture and association genetics. Nat Biotechnol. 2019;37(2):139-43.

3. Baggs E, Dagdas G, Krasileva KV. NLR diversity, helpers and integrated domains: making sense of the NLR IDentity. Curr Opin Plant Biol. 2017;38:59-67.

4. Bailey PC, Schudoma C, Jackson W, Baggs E, Dagdas G, Haerty W, et al. Dominant integration locus drives continuous diversification of plant immune receptors with exogenous domain fusions. Genome Biol. 2018;19:23.

5. Bailey $T L$, Gribskov M. Combining evidence using $p$-values: application to sequence homology searches. Bioinformatics. 1998;14(1):48-54.

6. Barbey CR, Lee S, Verma S, Bird KA, Yocca AE, Edger PP, et al. Disease resistance genetics and genomics in Octoploid strawberry. G3 (Bethesda). 2019;9(10):3315-32

7. Boller T, He SY. Innate immunity in plants: an arms race between pattern recognition receptors in plants and effectors in microbial pathogens. Science. 2009:324(5928):742-4.

8. Bozkurt O, Hakki EE, Akkaya MS. Isolation and sequence analysis of wheat NBS-LRR type disease resistance gene analogs using degenerate PCR primers. Biochem Genet. 2007:45(5-6):469-86.

9. Brabham HJ, Hernández-Pinzón I, Holden S, Lorang J, Moscou MJ. An ancient integration in a plant NLR is maintained as a trans-species polymorphism. BioRxiv. 2017. https://doi.org/10.1101/239541.

10. Brabham HJ, Hernandez-Pinzon I, Lorang JM, Wolpert TJ, Hayes PM, Sato $\mathrm{K}$, et al. Multiple pathogen recognition at the Mla locus in barley. Mol Plant-Microbe Interact. 2019;32(10):91-2.

11. Cao A, Xing L, Wang $X$, Yang $X$, Wang W, Sun $Y$, et al. Serine/threonine kinase gene $S t p k-V$, a key member of powdery mildew resistance gene Pm21, confers powdery mildew resistance in wheat. Proc Natl Acad Sci U S A. 2011;108(19):7727-32.

12. Cesari S, Bernoux M, Moncuquet P, Kroj T, Dodds PN. A novel conserved mechanism for plant NLR protein pairs: the "integrated decoy" hypothesis. Front Plant Sci. 2014;5:606.

13. Cesari S, Thilliez G, Ribot C, Chalvon V, Michel C, Jauneau A, et al. The Rice resistance protein pair RGA4/RGA5 recognizes the Magnaporthe oryzae effectors AVR-Pia and AVR1-CO39 by direct binding. Plant Cell. 2013;25(4):1463-81.
14. Chen PD, Qi LL, Zhou B, Zhang SZ, Liu DJ. Development and molecular cytogenetic analysis of wheat-Haynaldia villosa 6VS/6AL translocation lines specifying resistance to powdery mildew. Theor Appl Genet. 1995;91(6-7):1125-8.

15. Cheng X, Hang H, Zhao Y, Qian Y, Zhu S, Cheng B. A genomic analysis of disease-resistance genes encoding nucleotide binding sites in Sorghum bicolor. Genet Mol Biol. 2010;33(2):292-7.

16. Dai K, Zhao R, Shi M, Xiao J, Yu Z, Jia Q, et al. Dissection and cytological mapping of chromosome arm 4VS by the development of wheatHaynaldia villosa structural aberration library. Theor Appl Genet. 2020;133(1):217-26.

17. Dangl JL, Horvath DM, Staskawicz BJ. Pivoting the plant immune system from dissection to deployment. Science. 2013;341(6147):746-51.

18. Dodds PN, Rathjen JP. Plant immunity: towards an integrated view of plant-pathogen interactions. Nat Rev Genet. 2010;11 (8):539-48.

19. Giolai M, Paajanen P, Verweij W, Witek K, Jones JDG, Clark MD. Comparative analysis of targeted long read sequencing approaches for characterization of a plant's immune receptor repertoire. BMC Genomics. 2017;18:564.

20. Gradzielewska A. The genus Dasypyrum-part 2. Dasypyrum villosum-a wild species used in wheat improvement. Euphytica. 2006;152:441-54.

21. Grech-Baran M, Witek K, Szajko K, Witek Al, Morgiewicz K, Wasilewicz-Flis I, et al. Extreme resistance to potato virus $Y$ in potato carrying the Ry(sto) gene is mediated by a TIR-NLR immune receptor. Plant Biotechnol J. 2020;18(3):655-67.

22. Gu L, Si W, Zhao L, Yang S, Zhang X. Dynamic evolution of NBSLRR genes in bread wheat and its progenitors. Mol Gen Genomics. 2015;290(2):727-38.

23. Guo Y-L, Fitz J, Schneeberger K, Ossowski S, Cao J, Weigel D. Genomewide comparison of nucleotide-binding site-Leucine-rich repeat-encoding genes in Arabidopsis. Plant Physiol. 2011;157(2):757-69.

24. Huang L, Brooks S, LiW, Fellers J, Nelson JC, Gill B. Evolution of new disease specificity at a simple resistance locus in a crop-weed complex: reconstitution of the Lr21 gene in wheat. Genetics. 2009;182(2):595-602.

25. IBGSC. A physical, genetic and functional sequence assembly of the barley genome. Nature. 2012;491:711-6.

26. Jan CC, Depace C, McGuire PE, Qualset CO. Hybrids and amphiploids of Triticum aestivum L. and T.turgidum L., with Dasypyrum villosum (L.) Candargy. Z Pflanzenzüchtung. 1986;96(2):97-106.

27. Jia Y, Yuan $Y$, Zhang $Y$, Yang S, Zhang X. Extreme expansion of NBS-encoding genes in Rosaceae. BMC Genet. 2015;16:48.

28. Jones JDG, Dangl JL. The plant immune system. Nature. 2006:444(7117):323-9.

29. Jones JDG, Vance RE, Dangl JL. Intracellular innate immune surveillance devices in plants and animals. Science. 2016;354(6316):aaf6395.

30. Jones P, Binns D, Chang H-Y, Fraser M, Li W, McAnulla C, et al. InterProScan 5: genome-scale protein function classification. Bioinformatics. 2014;30(9):1236-40.

31. Jordan T, Seeholzer S, Schwizer S, Toeller A, Somssich IE, Keller B. The wheat Mla homologue TmMla1 exhibits an evolutionarily conserved function against powdery mildew in both wheat and barley. Plant J. 2011;65(4):610-21.

32. Jouanin A, Borm T, Boyd LA, Cockram J, Leigh F, Santos BACM, et al. Development of the GlutEnSeq capture system for sequencing gluten gene families in hexaploid bread wheat with deletions or mutations induced by gamma-irradiation or CRISPR/Cas9. J Cereal Sci. 2019;88:157-66.

33. Jupe F, Pritchard L, Etherington GJ, MacKenzie K, Cock PJA, Wright F, et al. Identification and localisation of the NB-LRR gene family within the potato genome. BMC Genomics. 2012;13:75.

34. Jupe F, Witek K, Verweij W, Sliwka J, Pritchard L, Etherington GJ, et al. Resistance gene enrichment sequencing (RenSeq) enables reannotation of the NB-LRR gene family from sequenced plant genomes and rapid mapping of resistance loci in segregating populations. Plant J. 2013;76(3):530-44.

35. Kang YJ, Kim KH, Shim S, Yoon MY, Sun S, Kim MY, et al. Genome-wide mapping of NBS-LRR genes and their association with disease resistance in soybean. BMC Plant Biol. 2012;12:139.

36. Kourelis J, van der Hoorn RAL. Defended to the nines: 25 years of resistance gene cloning identifies nine mechanisms for $\mathrm{R}$ protein function. Plant Cell. 2018;30(2):285-99.

37. Kroj T, Chanclud E, Michel-Romiti C, Grand X, Morel J-B. Integration of decoy domains derived from protein targets of pathogen 
effectors into plant immune receptors is widespread. New Phytol. 2016;210(2):618-26.

38. Kumar S, Stecher G, Tamura K. MEGA7: molecular evolutionary genetics analysis version 7.0 for bigger datasets. Mol Biol Evol. 2016;33(7):1870-4.

39. Lee SK, Song MY, Seo YS, Kim HK, Ko S, Cao PJ, et al. Rice Pi5-mediated resistance to Magnaporthe oryzae requires the presence of two coiled-coil-nucleotide-binding-leucine-rich repeat genes. Genetics. 2009:181(4):1627-38.

40. Letunic l, Bork P. Interactive tree of life (iTOL) v3: an online tool for the display and annotation of phylogenetic and other trees. Nucleic Acids Res. 2016;44(W1):W242-5.

41. Li J, Ding J, Zhang W, Zhang Y, Tang P, Chen JQ, et al. Unique evolutionary pattern of numbers of gramineous NBS-LRR genes. Mol Gen Genomics. 2010;283(5):427-38.

42. Li W, Godzik A. Cd-hit: a fast program for clustering and comparing large sets of protein or nucleotide sequences. Bioinformatics. 2006;22(13):1658-9.

43. Linde-Laursen I, Jensen HP, Jørgensen JH. Resistance of Triticale, Aegilops, and Haynaldia species to the take-all fungus, Gaeumannomyces graminis. Z Pflanzenzüchtung. 1973;70:200-13.

44. Liu J, Qiao L, Zhang X, Li X, Zhan H, Guo H, et al. Genome-wide identification and resistance expression analysis of the NBS gene family in Triticum urartu. Genes Genomics. 2017;39(6):611-21.

45. Liu W, Xie Y, Ma J, Luo X, Nie P, Zuo Z, et al. IBS: an illustrator for the presentation and visualization of biological sequences. Bioinformatics. 2015;31(20):3359-61.

46. Lozano R, Hamblin MT, Prochnik S, Jannink JL. Identification and distribution of the NBS-LRR gene family in the cassava genome. BMC Genomics. 2015;16:360.

47. Maekawa T, Kracher B, Vernaldi S, van Themaat EVL, Schulze-Lefert P. Conservation of NLR-triggered immunity across plant lineages. Proc Natl Acad Sci U S A. 2012;109(49):20119-23.

48. Maekawa T, Kufer TA, Schulze-Lefert P. NLR functions in plant and animal immune systems: so far and yet so close. Nat Immunol. 2011;12(9):818-26.

49. Mago R, Zhang P, Vautrin S, Simkova H, Bansal U, Luo MC, et al. The wheat Sr50 gene reveals rich diversity at a cereal disease resistance locus. Nat Plants. 2015;1(12):15186.

50. Meyers BC, Kozik A, Griego A, Kuang HH, Michelmore RW. Genomewide analysis of NBS-LRR-encoding genes in Arabidopsis. Plant Cell. 2003;15(4):809-34.

51. Mucyn TS, Clemente A, Andriotis VME, Balmuth AL, Oldroyd GED, Staskawicz BJ, et al. The tomato NBARC-LRR protein Prf interacts with Pto kinase in vivo to regulate specific plant immunity. Plant Cell. 2006:18(10):2792-806

52. Murray TD, Delapena RC, Yildirim A, Jones SS. A new source of resistance to Pseudocercosporella herpotrichoides, cause of eyespot disease of wheat, located on chromosome 4V of Dasypyrum villosum. Plant Breed. 1994;113(4):281-6.

53. Narusaka M, Shirasu K, Noutoshi Y, Kubo Y, Shiraishi T, Iwabuchi M, et al. RRS1 and RPS4 provide a dual resistance-gene system against fungal and bacterial pathogens. Plant J. 2009;60(2):218-26.

54. Neves LG, Davis JM, Barbazuk WB, Kirst M. Whole-exome targeted sequencing of the uncharacterized pine genome. Plant J. 2013;75(1):146-56.

55. Paulus JK, van der Hoorn RAL. Tricked or trapped-two decoy mechanisms in host-pathogen interactions. PLoS Pathog. 2018;14(2):e1006761.

56. Periyannan S, Moore J, Ayliffe M, Bansal U, Wang X, Huang L, et al. The gene Sr33, an Ortholog of barley Mla genes, encodes resistance to wheat stem rust race Ug99. Science. 2013;341(6147):786-8.

57. Pont C, Leroy T, Seidel M, Tondelli A, Duchemin W, Armisen D, et al. Tracing the ancestry of modern bread wheats. Nat Genet. 2019:51(5):905-11.

58. Porter BW, Paidi M, Ming R, Alam M, Nishijima WT, Zhu YJ. Genome-wide analysis of Carica papaya reveals a small NBS resistance gene family. Mol Gen Genomics. 2009;281(6):609-26.

59. Qi LL, Pumphrey MO, Friebe B, Zhang P, Qian C, Bowden RL, et al. A novel Robertsonian translocation event leads to transfer of a stem rust resistance gene (Sr52) effective against race Ug99 from Dasypyrum villosum into bread wheat. Theor Appl Genet. 2011;123(1):159-67.
60. Qian LH, Zhou GC, Sun XQ, Lei Z, Zhang YM, Xue JY, et al. Distinct patterns of gene gain and loss: diverse evolutionary modes of NBSencoding genes in three Solanaceae crop species. G3 (Bethesda). 2017;7(5):1577-85.

61. Rawal HC, Mithra SVA, Arora K, Kumar V, Goel N, Mishra DC, et al. Genome-wide analysis in wild and cultivated Oryza species reveals abundance of NBS genes in progenitors of cultivated Rice. Plant Mol Biol Rep. 2018;36(3):373-86.

62. Sanchez-Martin J, Steuernagel B, Ghosh S, Herren G, Hurni S, Adamski N, et al. Rapid gene isolation in barley and wheat by mutant chromosome sequencing. Genome Biol. 2016;17:221.

63. Sarris PF, Cevik V, Dagdas G, Jones JDG, Krasileva KV. Comparative analysis of plant immune receptor architectures uncovers host proteins likely targeted by pathogens. BMC Biol. 2016;14:8.

64. Shao ZQ, Xue JY, Wu P, Zhang YM, Wu Y, Hang YY, et al. Large-scale analyses of angiosperm nucleotide-binding site-Leucine-rich repeat genes reveal three anciently diverged classes with distinct evolutionary patterns. Plant Physiol. 2016;170(4):2095-109.

65. Sinapidou E, Williams K, Nott L, Bahkt S, Tor M, Crute I, et al. Two TIR:NB:LRR genes are required to specify resistance to Peronospora parasitica isolate Cala2 in Arabidopsis. Plant J. 2004;38(6):898-909.

66. Song W, Wang B, Li X, Wei J, Chen L, Zhang D, et al. Identification of immune related LRR-containing genes in maize (Zea mays $L$.) by genomewide sequence analysis. Int J Genomics. 2015;2015:231358.

67. Steuernagel B, Jupe F, Witek K, Jones DG J, Wulff BH B. NLR-parser: rapid annotation of plant NLR complements. Bioinformatics 2015;31(10):1665-7.

68. Steuernage B, Periyannan SK, Hernandez-Pinzon I, Witek K, Rouse MN, Yu G, et al. Rapid cloning of disease-resistance genes in plants using mutagenesis and sequence capture. Nat Biotechnol. 2016;34(6):652-5.

69. Steuernagel B, Witek K, Krattinger SG, Ramirez-Gonzalez RH, Schoonbeek H-j, Yu G, et al. The NLR-annotator tool enables annotation of the intracellular immune receptor repertoire(1)( OPEN ). Plant Physiol. 2020;183(2):468-82.

70. Thordal-Christensen $\mathrm{H}$. Fresh insights into processes of nonhost resistance. Curr Opin Plant Biol. 2003;6(4):351-7.

71. Van de Weyer A-L, Monteiro F, Furzer OJ, Nishimura MT, Cevik V, Witek K, et al. A species-wide inventory of NLR genes and alleles in Arabidopsis thaliana. Cell. 2019;178(5):1260-72.

72. van der Hoorn RAL, Kamoun S. From guard to decoy: a new model for perception of plant pathogen effectors. Plant Cell. 2008;20(8):2009-17.

73. Velasco R, Zharkikh A, Affourtit J, Dhingra A, Cestaro A, Kalyanaraman A, et al. The genome of the domesticated apple (Malus $x$ domestica Borkh.). Nat Genet. 2010;42(10):833-41.

74. Wang X, Richards J, Gross T, Druka A, Kleinhofs A, Steffenson B, et al. The rpg4-mediated resistance to wheat stem rust (Puccinia graminis) in barley (Hordeum vulgare) requires Rpg5, a second NBS-LRR gene, and an actin Depolymerization factor. Mol Plant-Microbe Interact. 2013;26(4):407-18

75. Wingen LU, West C, Leverington-Waite M, Collier S, Orford S, Goram R, et al. Wheat landrace genome diversity. Genetics. 2017;205(4):1657-76.

76. Witek K, Jupe F, Witek Al, Baker D, Clark MD, Jones JDG. Accelerated cloning of a potato late blight-resistance gene using RenSeq and SMRT sequencing. Nat Biotechnol. 2016;34(6):656-60.

77. Wulff BBH, Moscou MJ. Strategies for transferring resistance into wheat: from wide crosses to GM cassettes. Front Plant Sci. 2014;5:692.

78. Xiang L, Liu J, Wu C, Deng Y, Cai C, Zhang X, et al. Genome-wide comparative analysis of NBS-encoding genes in four Gossypium species. BMC Genomics. 2017;18:292.

79. Xing L, Hu P, Liu J, Witek K, Zhou S, Xu J, et al. Pm21 from Haynaldia villosa encodes a CC-NBS-LRR protein conferring powdery mildew resistance in wheat. Mol Plant. 2018;11(6):874-8.

80. Yang S, Feng Z, Zhang X, Jiang K, Jin X, Hang Y, et al. Genome-wide investigation on the genetic variations of rice disease resistance genes. Plant Mol Biol. 2006;62(1-2):181-93.

81. Yang S, Zhang X, Yue JX, Tian D, Chen JQ. Recent duplications dominate NBS-encoding gene expansion in two woody species. Mol Gen Genomics. 2008;280(3):187-98.

82. Zhai C, Zhang Y, Yao N, Lin F, Liu Z, Dong Z, et al. Function and interaction of the coupled genes responsible for Pik-h encoded Rice blast resistance. PLoS One. 2014;9(6):e98067. 
83. Zhang R, Fan Y, Kong L, Wang Z, Wu J, Xing L, et al. Pm62, an adultplant powdery mildew resistance gene introgressed from Dasypyrum villosum chromosome arm 2VL into wheat. Theor Appl Genet. 2018;131(12):2613-20.

84. Zhang R, Feng Y, Li H, Yuan H, Dai J, Cao A, et al. Cereal cyst nematode resistance gene CreV effective against Heterodera filipjevi transferred from chromosome 6VL of Dasypyrum villosum to bread wheat. Mol Breed. 2016;36(9):122.

85. Zhang R, Sun B, Chen J, Cao A, Xing L, Feng Y, et al. Pm55, a developmental-stage and tissue-specific powdery mildew resistance gene introgressed from Dasypyrum villosum into common wheat. Theor Appl Genet. 2016;129(10):1975-84.

86. Zhang $R$, Yao R, Sun $D$, Sun B, Feng $Y$, Zhang $W$, et al. Development of $V$ chromosome alterations and physical mapping of molecular markers specific to Dasypyrum villosum. Mol Breed. 2017;37(5):67.

87. Zhang X, Wei X, Xiao J, Yuan C, Wu Y, Cao A, et al. Whole genome development of intron targeting (IT) markers specific for Dasypyrum villosum chromosomes based on next-generation sequencing technology. Mol Breed. 2017;37(9):115.

88. Zhang YM, Shao ZQ, Wang Q, Hang YY, Xue JY, Wang B, et al. Uncovering the dynamic evolution of nucleotide-binding site-leucine-rich repeat (NBS-LRR) genes in Brassicaceae. J Integr Plant Biol. 2016;58(2):165-77.

89. Zheng F, Wu H, Zhang R, Li S, He W, Wong FL, et al. Molecular phylogeny and dynamic evolution of disease resistance genes in the legume family. BMC Genomics. 2016;17:402.

90. Zhou Y, Zhao X, Li Y, Xu J, Bi A, Kang L, et al. Triticum population sequencing provides insights into wheat adaptation. Nat Genet. 2020;52:1412-22.

\section{Publisher's Note}

Springer Nature remains neutral with regard to jurisdictional claims in published maps and institutional affiliations.

- fast, convenient online submission

- thorough peer review by experienced researchers in your field

- rapid publication on acceptance

- support for research data, including large and complex data types

- gold Open Access which fosters wider collaboration and increased citations

- maximum visibility for your research: over $100 \mathrm{M}$ website views per year

At BMC, research is always in progress.

Learn more biomedcentral.com/submissions 